Успехи физ. мет. / Usp. Fiz. Met. 2005, т. 6, сc. 1-54 Оттиски доступны непосредственно от издателя Фотокопирование разрешено только в соответствии с лицензией (c) 2005 ИМФ (Институт металлофизики им. Г. В. Курдюмова НАН Украины)

Напечатано в Украине.

PACS numbers: 05.40.Ca, 05.45.Df, 05.65.+b, 05.70.Fh, 45.70.Ht, 64.60.Ak

\title{
Self-Organization of Condensed Matter in Fluctuating Environment
}

\author{
D. O. Kharchenko
}

Sumy State University,
2 Ryms'ky-Korsakov Str.,
UA-40007 Sumy, Ukraine

A synergetic conception of plastic deformation is presented allowing for the defects' interplay resulting in a phase transition. A qualitative-reconstruction pattern of imperfect condensed-matter structure in the presence of fluctuations in both 0-dimensional and distributed (extended) systems is considered. As shown, the system undergoes the reversible phase transitions under the increasing intensity of fluctuations, diffusion constituent and deformation-type external parameters. Within the framework of the synergetic approach based on the Lorenz system, a pattern of explosive crystallization is shown to be scripting the same way as the avalanches formation. The relationships are set between the exponent of a size distribution of avalanches, the fractal dimension of a phase space, characteristics of noise, the number of governing equations, the dynamical exponent, and the non-additivity parameter. As shown, the regime of self-organized criticality is associated with an anomalous diffusion processes.

Представлено синергетичну концепцію пластичної деформації із урахуванням взаємодії між дефектами, в рамках якої досліджуються фазові переходи. Розглянуто картину якісної перебудови структури конденсованого середовища при наявності флюктуацій у 0-вимірних та розподілених системах. Показано, що система здатна зазнавати реверсивні переходи із зростанням інтенсивности флюктуацій, дифузійної складової та зовнішніх параметрів типу деформації. В рамках синергетичного підходу на основі Льоренцової системи розглянуто картину вибухової кристалізації, яка споріднена процесам формування лавин. 3 'ясовано співвідношення між показником в розподілі за розмірами лавин, фрактальною вимірністю фазового простору, характеристиками флюктуацій, числом керуючих рівнань, динамічним показником, параметром неадитивности. Показано, що режим самоорганізованої критичности тісно пов'язаний із процесами аномальної дифузії.

Представлена синергетическая концепция пластической деформации, 
учитывающая взаимодействия между дефектами, в рамках которой исследуются фазовые переходы. Рассмотрена картина качественной перестройки структуры конденсированной среды при наличии флуктуаций в 0-мерных и распределенных системах. Показано, что система способна претерпевать реверсивные фазовые переходы с возрастанием интенсивности флуктуаций, диффузионной составляющей и внешних параметров типа деформации. В рамках синергетического подхода на основе системы Лоренца рассмотрена картина взрывной кристаллизации, протекающей по сценарию процессов лавинообразования. Установлены соотношения между показателями распределения лавин по размерам, фрактальной размерностью фазового пространства, характеристиками флуктуаций, числом управляющих уравнений, динамическим показателем и параметром неаддитивности. Показано, что режим самоорганизованной критичности тесно связан с процессами аномальной диффузии.

Key words: noise-induced phase transitions, order parameter, self-organized criticality, fractal dimension, anomalous diffusion.

(Received April 6, 2004)

\section{INTRODUCTION}

After years of introduction of the synergetics to describe the complex behaviour of nonlinear systems [1,2], the attention of researchers started to drift toward the nonequilibrium statistical ensembles, which display the coherent behaviour caused by the collective effects. Along this line the deterministic approaches to study complex systems were enriched by the engaging the stochastic methods of analysis [3, 4]. It allows finding nontrivial peculiarities of nonequilibrium phase transitions in noisy spatially extended systems, which cannot be discovered by ignoring the fluctuations of environment [5]. As was displayed in a last decade, among the phase transitions that occur only when a control parameter is driven above a critical value one has another qualitative change of the system behaviour associated with the regime of selforganized criticality [6, 7]. In such a regime, a spontaneous (avalanchetype) dynamics is observed. A main feature of the systems displaying self-organized criticality is their self-similarity corresponded to fractal properties of the system and manifested itself in laws of motion [8, 9].

All mentioned features of the complex behaviour of nonlinear systems could be found considering a condensed matter where an atoms' system is moved off the equilibrium state. Such a situation is realized in the kernel of defect of crystal lattice or in domains of plastic flow and destruction. It is well known that the plastic flow of polycrystalline materials is a complex many-stage process, which occurs on different structure levels being characterized by its own scale and a type of structure element $[10,11]$. In the course of plas- 
tic deformation, the nonlinear coupling between the elements of structure of one type and elements of different structure levels is realized. It results to the complex cooperative behaviour of the system of defects such as appearing regular spatial-temporal structures on a macroscale level: the chaotic structure of dislocations is transformed to the dislocation tangles and cell boundaries, which can be transformed to the strip structure. Above-mentioned transformations of defect structure can be represented on the basis of conception of nonequilibrium phase transitions. A special attention can be paid considering the explosive crystallization process [12] relative to the regime of self-organized criticality. Here, a small number of embryos of crystallization initiate the crystallization of amorphous films over whole surface during a small time interval. Such a system shows the hierarchical structure, and hence it is characterized by fractal properties.

Main purpose of this contribution is to present such peculiarities of complex behaviour of defect structure in the framework of unique conception for far off-equilibrium systems. It is reached on the basis of synergetic scheme representing an evolution of hydrodynamic modes parametrizing the system. We re aimed to render the theoretical analysis of the synergetic systems in fluctuation environment. Section 2 is devoted to development of contemporary representations of transition from stochastic regime to coherent one. In Section 2.1, we present most popular models of plastic flow and reconstruction of defect structure: the 'activator-inhibitor' model shown in terms of deformation and tension (Section 2.1.1) and Lorenz-type scheme to describe a formation of strip of localized plastic flow (Section 2.1.2). Section 2.2 is devoted to consideration of the phase transitions induced by fluctuations of linear defects density and external stress (strain). In Section 2.2.1, we present a phenomenological model to account the evolution of defects with different dimensionalities in the field of plastic deformation on the basis of the assumptions of 'predator-prey' model. We show the correlated fluctuations stimulate the re-entrant noise-induced phase transitions familiar to the formation of different types of defect structure (Section 2.2.2). In Section 2.2.3, we consider the effect of correlations between fluctuations of system parameters. We demonstrate that this cross correlations can be a reason of the formation of different types of defect structures. As will be shown, the ordered state appears in the course of a chain of phase transitions. Moreover, we will show that different ordered structures arise as a result of the first- and second-order phase transitions.

Section 3 deals with the investigation of the systems where the avalanche-like dynamics is observed. In condensed matter, such behaviour is realized in the process of explosive crystallization. In 
Section 3.1, we present the mechanisms of process of explosive crystallization in which the formation of avalanche is possible. The experimental data is discussed in Section 3.1.1. The analytic description of explosive crystallization in the framework of the Lorenz-type model is shown in Section 3.1.2. We treat the transition to such a regime as the first order phase transition where the velocity of crystallization sharply increases from zero to the fixed magnitude. The deterministic picture of such a transition assumes the special form of relaxation process for the velocity of crystallization. However, such approach does not allow describing the distribution over the avalanche size or over the velocity. The simplest approach to account the first-order transition and to obtain above distribution is achieved considering the fluctuations of main modes of the model (Section 3.2). To this end, we introduce a unified Lorenz system with a fractional feedback and consider the noise-induced transitions to obtain the phase diagram giving the domain in which the regime of self-organized criticality is realized. The above generalization puts forward basis of Section 3.2.1 devoted to consideration of avalanche ensemble. Following famous Edwards paradigm [13, 14], an effective scheme addressed to the nonextensive thermodynamics [15] is proposed to determine a time-dependent distribution over energies of equipped sites in the explosive crystallization process. Generalization of the Edwards scheme to nonstationary nonextensive systems is reached on the basis of the fractional Lorenz system where the avalanche size plays a role of the order parameter, nonextensive complexity is reduced to the conjugate field and the nonconserved energy of the equipped sites is the control parameter. Within the framework of this approach, the phase diagram is calculated to define the different domains of system behaviour as a function of noise intensities of the above values. As a result, we arrive at a natural conclusion that the power-law distribution inherent in the self-organized criticality regime is caused by noise of the energy. In Section 3.2.2, we show that this distribution is the solution of both nonlinear Fokker-Planck equation, which appears in the description of nonextensive systems [15], and fractional FokkerPlanck equation inherent in Lévy-type processes [16]. We obtain relations between the main exponents of self-organized criticality regime, dynamical and geometry exponents of the model. Finally, main results are discussed in conclusion.

Appendix contains the basic properties of fractional integral and derivative.

\section{COOPERATIVE BEHAVIOUR OF DEFECTS' ENSEMBLE}

As well known, the irreversible reconstruction of crystal structure 
involves a permanent deformation process. Such a phenomenon is known as a plastic deformation. At weak deformation, the description of processes of plastic flow can be reached in the framework of typical scheme of evolution of ensemble of plastic deformation carriers in external field [17-23]. In such a case, the density of defects is a small and they behave themselves independently: in the course of plastic flow, defects obey the action of external field and interaction forces between them. When the deformation is increased, the density of carriers becomes so large that the assumption of autonomous behaviour of defects is failed [11]. In such an approach, it is necessary to consider a coherent behaviour of defects whose evolution should be examined. A transition from an autonomous regime to a coherent one results in formation of stripe of plastic flow due to autocatalytic generation of dislocations and vacancies or interstitial atoms. The transition to the coherent regime is a complex process where different defect structures are observed. In the course of plastic deformation, a strong linkage between elements of different defect structures is realized. It results in appearance of regular spatial-temporal structures on a macroscopic scale. For instance, an initially homogeneous crystallite is transformed to a weak offoriented cellular structure, which transforms into a fragmented structure at large deformation magnitudes [11, 24] (see Fig. 1).

A mechanism of generation and interaction of defects can be the following: (i) an interaction between dislocations of different sliding systems; (ii) formation of generating thresholds, which produce dipole configurations of dislocations; (iii) generation of atom defects as a climb of edge component of elementary thresholds. Point defects themselves have not influence on the plastic behaviour of crystal directly, but their annihilation results in edge dislocations creation. On the other hand, a coupling point defects with dislocations derives to the plastic deformation contribution with the deformation velocity being proportional to production of concentrations of linear and point defects.

To formulate a related scheme we give the most popular synergetic models (Section 2.1) to study the plastic deformation and to treat some special properties of this process. The two-parameter approach based on the 'activator-inhibitor' model is considered in Section 2.1.1. Here, the role of 'activator' is played by the deformation, whereas a tension is an 'inhibitor' to be a conjugate parameter. A collective mode of linear defects in the course of plastic deformation is studied in Section 2.1.2. We show that such a model has a form of the Lorenz-type system, which is the simplest way to consider a self-organization of defect structure. In Section 2.1.3, we present the model to account the evolution of defects with different dimensionalities in the framework of three-parameter synergetic 

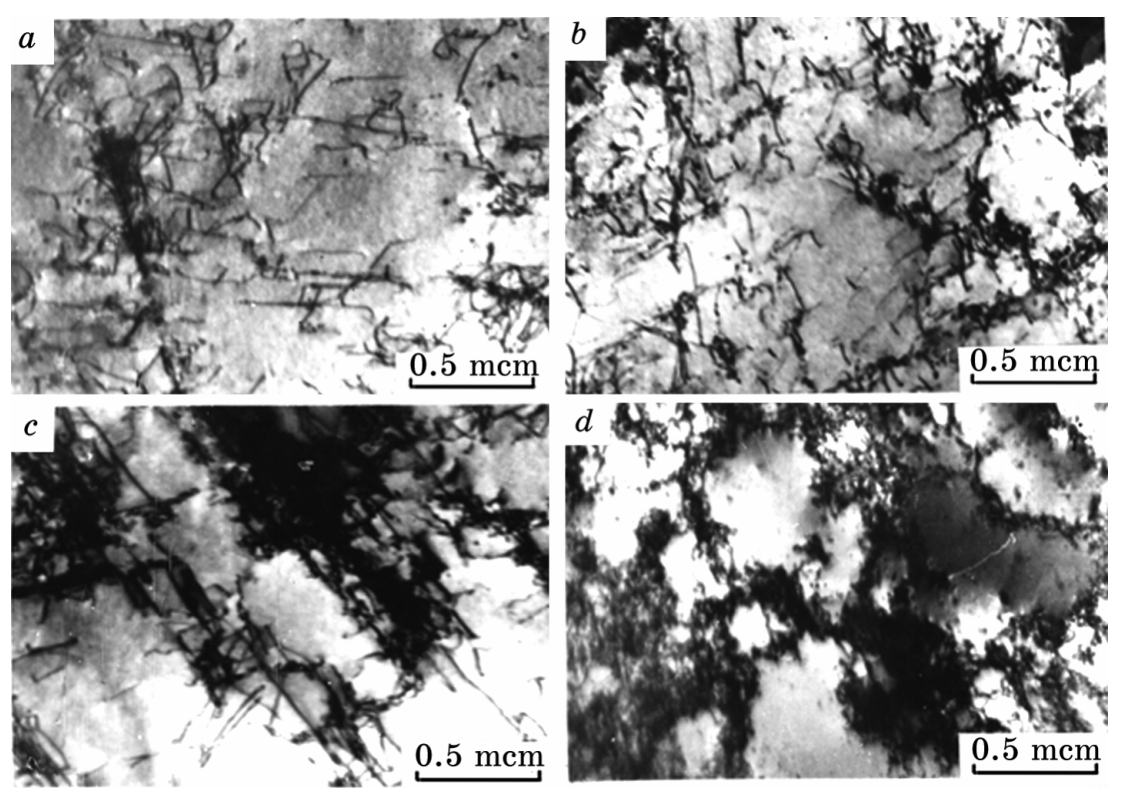

Fig. 1. Electron microscope views of dislocation structure of ordered $\mathrm{Ni}_{3} \mathrm{Fe}$ alloys for differential strains $(a-d$ corresponds to deformation $\theta=0.05$, $0.05,0.16,0.28)[24]$.

conception. The noisy system is considered in Section 2.2. As shown, the noise induces phase transitions corresponding to the rebuilding defect structure in the course of deformation process. The systems with one and two noises are considered in Sections 2.2.1 and 2.2 .2 respectively.

\subsection{SYNERGETIC CONCEPTION OF PLASTIC DEFORMATION}

The developing the domain of localized deformation results to the regime where a relaxation processes are realized. It means that the picture of kinetics of defects ensemble is relevant to the corresponding dynamical problem consideration. Such a situation is realized at large enough values of density of defects whose coherent evolution results in collective effects, which cannot be considered in usual way. To perform the adequate description one needs to pass to the synergetic scheme to represent time dependences of density of coherent ensemble of deformation carriers in the field of deformation.

\subsubsection{Activator-Inhibitor Model}

The one of the simplest approach to study the process of plastic de- 
formation is the using the two-component 'activator-inhibitor' model where the deformation $\varepsilon$ plays a role of an activator and the shear component $\tau$ of the tension is an inhibitor [1]. Such a model allows one to consider the inhomogeneity of deformation process. By this, the autocatalytic factor is deformation to be stimulus for the process where every shear step initiates the corresponding process in a neighbour domain with characteristic length $\ell$, and the deformation rate is comparable with the velocity of dislocation motion $v_{d}$. On the other hand, at each act of shearing process the elastic energy is redistributed over the bulk by means of elastic wave of acoustic emission to produce a tension decrease. The last results in damping process that has an effective radius comparable with a size of the system $L>>\ell$ and a rate of order of the velocity of elastic waves $v_{s} \gg v_{d}$ ( $v_{s}$ is the sound velocity). If we assume the environment, where the plastic deformation occurs, is of a mosaic of differently deformed domains, then we can suppose that deformation is a function of time and space, i.e. $\varepsilon=\varepsilon(t, \mathbf{r})$.

To find related field equation we suppose the deformation $\varepsilon=\varepsilon_{e}+\varepsilon_{v}$ is composed in elastic $\varepsilon_{e}$ and viscous $\varepsilon_{v}$ components. According the definition of the former, we have $\dot{\varepsilon}_{v}=v \Delta \varepsilon_{v}$, where $v$ is a kinematic-viscosity coefficient. As a result, we derive to the law of plastic flow as a diffusion process:

$$
\dot{\varepsilon}=f(\varepsilon, \tau)+v \Delta \varepsilon,
$$

where $f(\varepsilon, \tau)$ - a generalized force. In a similar manner, we can postulate the law of motion for a tension field

$$
\dot{\tau}=g(\varepsilon, \tau)+D_{\tau} \Delta \tau .
$$

where $g(\varepsilon, \tau), D$ stand for generalized force and inhomogeneous coefficient respectively. It is easy to see the estimations $v \sim v_{d} \ell$, $D \sim v_{s} L$. Further, our consideration reduces to definition of explicit forms for generalized forces- $f(\varepsilon, \tau), g(\varepsilon, \tau)$.

\subsubsection{Lorenz-Type Model}

The approach given by equations (2.1) does not take into account the dislocation mechanism of plastic deformation. At large densities of dislocations $\rho$ the incoherent coupling causes appearing the collective component $\rho_{c o l} \leq \rho$, which behaves itself in autonomous regime. Here, the interaction fields of dislocations are became of order of external tension [10] and a long-life hydrodynamic mode arises with relaxation time $t_{d} \gg \omega_{D}^{-1} \cong 10^{-13} \mathrm{~s}\left(\omega_{D}\right.$ is the Debye frequency) and amplitude $d \sim b \sqrt{\rho_{c o l}}$. In autonomous regime, the re- 
laxation of this mode is described by the Debye law $\dot{d}=-d / t_{d}$. However, the coherent coupling between defects causes a positive feedback between the tension and the deformation to appear the term being proportional to $\tau \varepsilon$. Accordingly to the Debye equation for the tension, $\dot{\tau}=\left(\tau_{e}-\tau\right) / t_{\tau}$, which relaxes to the stationary value $\tau_{e} \neq 0$, the negative feedback $\varepsilon d$ of mode of defects in the field of deformation is added. To construct the complete system of equations we take into account the Maxwell equation for the viscoelastic matter. As a result, we arrive at the following Lorenz-type system [25]:

$$
\begin{aligned}
& \dot{\tau}=\left(\tau_{e}-\tau\right) / t_{\tau}-g_{\tau} \varepsilon d, \\
& \dot{d}=-d / t_{d}+g_{d} \tau \varepsilon, \\
& \dot{\varepsilon}=-\varepsilon / t_{\varepsilon}+g_{\varepsilon} d .
\end{aligned}
$$

For the sake of simplicity, we consider here the homogeneous model and put $D_{\varepsilon}=D_{\tau}=0$. The scales of the relaxation times $t_{\tau}, t_{d}, t_{\varepsilon}$ are as follows: for tension relaxation time one has $t_{\tau} \sim \omega_{D}^{-1}$; the relaxation time of collective mode $t_{d}$ is defined by means of the thermoactivated processes of migration, so that $t_{d} \sim \omega_{D}^{-1} \exp (Q / T)$ where $Q$ is the energy of activation, $T$ is the temperature; the relaxation time of deformation is $t_{\varepsilon}=\eta / \mu$, where $\eta$ and $\mu$ are a shear viscosity and an elasticity modulus. It allows treating the deformation $\varepsilon$ as the slowest variable. Within the synergetic scheme, the slowest mode is known as an order parameter, $d$ plays a role of conjugate field, and the tension $\tau$ corresponds to a control parameter. According to the relation

$$
t_{\varepsilon}>t_{d}, t_{\tau},
$$

one can use the adiabatic elimination procedure. It allows reducing the number of evolution equations and passing from the dynamical system (2.2) to the one-parameter equation

$$
t_{\varepsilon} \dot{\varepsilon}=-\frac{\partial V(\varepsilon)}{\partial \varepsilon},
$$

where the synergetic potential reads

$$
V(\varepsilon)=\frac{\varepsilon^{2}}{2}\left[1-\theta\left(\frac{\varepsilon}{\varepsilon_{m}}\right)^{-2} \ln \left(1+\left(\frac{\varepsilon}{\varepsilon_{m}}\right)^{2}\right)\right], \theta \equiv \tau_{e} g_{\varepsilon} g_{d}, \varepsilon_{m} \equiv \frac{1}{\sqrt{t_{d} t_{\tau} g_{d} g_{\tau}}} .
$$

Respectively, the values of $\tau$ and $d$ are given by the expressions:

$$
\tau=\frac{\tau_{e}}{1+\left(\varepsilon / \varepsilon_{m}\right)^{2}}, \quad d=t_{d} g_{d} \tau_{e} \frac{\varepsilon}{1+\left(\varepsilon / \varepsilon_{m}\right)^{2}} .
$$


It can be shown that the potential (2.5) is addressed to a secondorder phase transition. Indeed, the form of $V(\varepsilon)$ depends on the tensor of external tension $\sigma_{e x t}$ which defines the stationary value $\tau_{e}\left(\sigma_{e x t}\right)$ of shear component of internal tensions. At values $\sigma_{e x t}$ providing $\theta<1$, the potential (2.5) monotonically increases from the minimum $\varepsilon_{0}=0$. It means the relaxation to the state with $\varepsilon_{0}=0$ where the collective behaviour of defect ensemble has no effect on the value of plastic deformation. Physically, the small values of $\theta$ correspond to the relaxation and strengthening processes. At $\theta>1$, the potential (2.5) acquires minimum at nonzero value $\varepsilon_{0}=\varepsilon_{m}(\theta-1)^{1 / 2}$. It means appearing the plastic deformation caused by the coherent behaviour of ensemble of dislocations and vacancies. An effective time $t_{\text {eff }}=t_{\varepsilon}(\theta-1)^{-1}$ of passage into the coherent regime increases monotonically at $\theta \rightarrow 1$.

The main results of above scheme are as follows: (i) autocatalytic generation of defects causes the coherent coupling, and defects behave themselves in the collective manner in course of the time $t \sim t_{\text {eff }}$ under effect of external tension $\tau_{e}\left(\sigma_{e x t}\right)$; (ii) the deformation $\varepsilon$ defined through the autonomous defects acquires contribution, which increases in course of time $t>t_{\text {eff }}$ to the stationary value $\varepsilon_{0} \neq 0$. The proposed scheme can be generalized obviously through the consideration of the stripe of localized plastic deformation that requires accounting the spatial distribution $\varepsilon(\mathbf{r})$ formed at $t>t_{\text {eff }}$.

\subsection{NOISE-INDUCED PHASE TRANSITIONS}

\subsubsection{Evolution of Defects' Ensemble with Different Dimensionalities}

This model generalizes the well-known 'predator-prey' model where a role of 'predator' is played by dislocations, and the point defects serve as 'prey'. Moreover, we introduce the deformation as a control parameter in addition to above parameters. The main assumption is the linear form of equations for the density of dislocations $\rho$ and density of the point defects $c$. In these equations, we account the Debye relaxation processes for both the order parameter and conjugate field.

It will be convenient to introduce the order parameter (hydrodynamic mode amplitude) being the collective mode $d$. According to the assumption that dislocation loops are generated through the annihilation of point defects, we set an equation for the relative density of dislocations in the form

$$
t_{d} \dot{d}=-d+\alpha c .
$$

Here, $t_{d}$ is the relaxation time, $\alpha$ is the intensity of the generating the dislocation loops through the transformation of point defects ensemble. An equation for the point defects is as follows 


$$
t_{c} \dot{c}=-c+\beta d,
$$

where $t_{c}$ is the relaxation time for point-defects' concentration, $\beta$ is a function of the deformation $\varepsilon$ to be measure of the intensity of the point defects generation due to the climb of the dislocation thresholds in the field of plastic deformation. An equation for the deformation $\varepsilon$ should contain the relaxation term to define a decrease in the deformation to the nonzero value $\varepsilon_{0}$, i.e. $t \dot{\varepsilon}=\varepsilon_{0}-\varepsilon$. Moreover, we should take into account the term $\dot{\varepsilon} \cong \kappa c d$ of the deformation velocity given by the flow of defects produced in the process of plastic deformation. The proportionality coefficient $\kappa=\kappa(\Omega \tau / T)$ depends on the atom volume $\Omega$, the temperature $T$ and tension $\tau$. Next, we suppose that the deformation occurs in such a way that assumption $\kappa=$ const is satisfied. Combining corresponding expressions, we find the equation for the control parameter in the form:

$$
t_{\varepsilon} \dot{\varepsilon}=\varepsilon_{0}-\varepsilon+\kappa c d .
$$

Equations (2.7)-(2.9) are complete set to describe the evolution process of defect structure in the field of plastic deformation. One remark should be done about the deformation dependence of the coefficient $\beta$ in Eq. (2.8). Indeed, there is the nonlinearity in the third equation to be a reason for the self-organization in the system. Such a positive feedback should be compensated by a negative feedback in the second equation as a manifestation of the Le Chatelier principle. For this purpose, we expand $\beta$ in series up to the first order terms: $\beta \cong \beta_{0} \varepsilon_{0}-\beta_{1} \varepsilon$ with $\beta_{0}>\beta_{1}>0$. Then, with accounting the diffusion character of the evolution of the defects and Eq. (2.1a), we get finally

$$
\begin{aligned}
& t_{d} \dot{d}=-d+\alpha c+\ell_{d}^{2} \Delta d, \\
& t_{c} \dot{c}=-c+\left(\beta_{0} \varepsilon_{0}-\beta_{1} \varepsilon\right) d+\ell_{c}^{2} \Delta c, \\
& t_{\varepsilon} \dot{\varepsilon}=\varepsilon_{0}-\varepsilon+\kappa c d+\ell^{2} \Delta \varepsilon,
\end{aligned}
$$

where $\ell_{d}, \ell_{c}$ are corresponding diffusion lengths and $\ell$ is a characteristic length of deformation field variation. The last term in first equation reflects processes of interactions of dislocations. It is physically important that the obtained system (2.10) incorporates the defect densities with different dimensionalities.

Considering system with hierarchical slaving inhomogeneities for which $\ell_{d} \gg \ell_{c}, \ell$ and making use of the adiabatic elimination procedure where $t_{\varepsilon}, t_{c}<<t_{d}$, we derive to the equation for dimensionless order parameter $x=d / d_{m}\left(d_{m}^{2}=1 / \beta_{1} \kappa\right)$ in the form 


$$
\begin{gathered}
t_{d} \frac{d}{d t} x=-\frac{\partial V(x)}{\partial x}+\ell_{d}^{2} \Delta x, V(x)=\frac{1}{2}\left[x^{2}-\theta \ln \left(1+x^{2}\right)\right], \\
\theta \equiv \alpha\left(\beta_{0}-\beta_{1}\right) \varepsilon_{0}, \beta_{0}>\beta_{1} .
\end{gathered}
$$

With accuracy of the inhomogeneity term, this equation has the form of the Lorenz-type equations (2.4), (2.5). It was shown the stationary magnitude of the order parameter $x_{0}$ monotonically increases in the course of plastic deformation as $x_{0}=(\theta-1)^{1 / 2}$. Such a situation corresponds to the well-known experimental data for the scalar density of dislocation vs. deformation. However, the model (2.10) predicts an increasing the dislocation density only and does not give an information about reconstruction of defect structure. In reality, there are transitions from chaotic configuration to the tangles and then to a cellular structure. In the cellular structure, we have dislocation clusters with different directions of the Burgers vector $b$. To find the distribution of the clusters we consider spatial-temporal evolution of defect structure and pass to a study of the statistical system. Next, we are aimed to show correlated fluctuations in the system parameters is one of the reasons of rebuilding the defect structure in the course of the plastic deformation process.

To account the stochastic effects the model (2.10) should be rewritten in the following form:

$$
\begin{aligned}
& t_{d} \dot{d}=-d+\alpha c+\ell_{d}^{2} \Delta d+\sigma_{d} \zeta_{d}(r, t), \\
& t_{c} \dot{c}=-c+\left(\beta_{0} \varepsilon_{0}-\beta_{1} \varepsilon\right) d+\ell_{c}^{2} \Delta c+\sigma_{c} \zeta_{c}(r, t), \\
& t_{\varepsilon} \dot{\varepsilon}=\varepsilon_{0}-\varepsilon+\kappa c d+\ell^{2} \Delta \varepsilon+\sigma_{\varepsilon} \zeta_{\varepsilon}(r, t) .
\end{aligned}
$$

Here, stochastic terms $\zeta_{d}, \zeta_{c}, \zeta_{\varepsilon_{2}}$ define internal fluctuations of the main modes with intensities $\sigma_{d}^{2}, \sigma_{c}^{2}, \sigma_{\varepsilon}^{2}$. For the sake of simplicity, we assume the stochastic forces are Gaussian distributed with average $\left\langle\zeta_{\mu}(\mathbf{r}, t)\right\rangle=0$ and correlation functions

$$
\left\langle\zeta_{\mu}(\mathbf{r}, t) \zeta_{v}\left(\mathbf{r}^{\prime}, t^{\prime}\right)\right\rangle=C_{\mu v}\left(\left|t-t^{\prime}\right|\right) \delta\left(\mathbf{r}-\mathbf{r}^{\prime}\right), \mu, v=\{d, c, \varepsilon\} .
$$

In other words, we consider the time correlations only neglecting space correlations. Because the consideration of heterogeneity of main modes derives to a complex problem, we address the dislocations contribution only. This means, as in Eq. (2.11), the condition of $\ell_{d}>\ell_{c}, \ell$.

At passage to consideration of stochastic terms in system (2.12), it should be noted the simplest picture is related to white noise approach. It means the Fourier components of these terms are frequency independent. However, the real physical picture is characterized further by coloured fluctuations whose spectrum incorporates an assigned fre- 
quency domain. The simplest model to study such fluctuations is the Ornstein-Uhlenbeck process related to stochastic equation in the form

$$
\tau_{\mu}^{a} \dot{\zeta}_{\mu}=-\zeta_{\mu}+\xi_{\mu}(t), \mu=\{d, c, \varepsilon\},
$$

where $\tau_{i}^{a}$ is an autocorrelation time for the initial process $\zeta_{\mu}(t)$, whereas $\xi(t)$ is a white noise with following properties: $\left\langle\xi_{\mu}(t)\right\rangle_{\mu}=0$, $\left\langle\xi_{\mu}(t) \xi_{v}\left(t^{\prime}\right)\right\rangle=\delta_{\mu, v} \delta\left(t-t^{\prime}\right)$. According to Eq. (2.14), the correlation functions of coloured noises $\zeta_{\mu}(t)$ are as follows:

$$
C_{\mu, v}\left(t, t^{\prime}\right)=\frac{\sigma_{\mu} \sigma_{v}}{\tau_{\mu, v}^{c}} \exp \left(-\frac{\left|t-t^{\prime}\right|}{\tau_{\mu, v}^{c}}\right),
$$

where $\tau_{\mu, v}^{c}$ is the cross correlation time between the noises $\zeta_{\mu}$ and $\zeta_{v}$. At $\mu=v$, correlation times $\tau_{\mu, v}^{c}$ are reduced to the autocorrelation times $\tau_{\mu, \mu}^{a}$.

Following the assumption that the density of dislocations is a slow mode, we suppose $t_{c} \dot{c} \cong 0, t_{\varepsilon} \dot{\varepsilon} \cong 0$, and $\ell_{c}^{2} \Delta c=\ell^{2} \Delta \varepsilon=0$. Hence, Eqs. (2.12) yield

$$
\dot{x}=f(x)+D \Delta x+\sum_{\mu} g_{\mu}(x) \zeta_{\mu}(t)
$$

where the deterministic force and multiplicative functions are

$$
f(x)=-\frac{\partial V(x)}{\partial x}, g_{x}=1, g_{c}(x)=\left(1+x^{2}\right)^{-1}, g_{\varepsilon}(x)=x g_{c}(x) ;
$$

the form of the potential $V$ is given by Eq. (2.11)

To explore the picture of noise induced phase transitions, we represent the equation (2.16) in the regular $d$-dimension lattice of mesh size $\Delta l=1$. Then states of the system will be determined by a set of scalar variables $\left\{x_{k}\right\}, k=1, \ldots, L^{d}$, defined on the hypercubic grid of the size $L$. Making use of this approach the evolution of $\left\{x_{k}\right\}$ is defined through the set of equations

$$
\dot{x}_{k}=f\left(x_{k}\right)+\frac{D}{2 d} \sum_{j} D_{i j} x_{j}+\sum_{\mu} g_{\mu}\left(x_{k}\right) \zeta_{\mu, k}(t)
$$

where the definition of the Laplacian operator on the grid is used

$$
\sum_{j} D_{i j}=\sum_{j \in n n(k)}\left(\delta_{n n(j)}-2 d \delta_{k j}\right)
$$

with $n n(k)$ being a nearest neighbours of the site $k$.

It is appeared the diffusion can be represented as an effective interaction of nearest neighbours. Indeed, let's introduce the harmonic energy of such interactions by the potential 


$$
V_{\text {int }}=\frac{a}{2} \sum_{j}\left(x-x_{j}\right)^{2}
$$

where $a$ is the rigidity of effective spring and the sum is carried out over the locations $x_{j}$ of the nearest neighbours. In the framework of the mean field approximation $x_{j}$ is replaced by the mean value $\eta \equiv<x_{j}>$ after which the interaction force $f_{i n t}=-\partial V_{i n t} / \partial x$ acquires the form

$$
f_{\text {int }}=-D(x-\eta)
$$

where we have introduced the characteristic interaction coefficient $D=a z$ ( $z$ is a number of nearest neighbours). Then, we obtain the equation (space subscripts are dropped)

$$
\dot{x}=f(x)+f_{i n t}(x ; \eta)+\sum_{\mu} g_{\mu}(x) \zeta_{\mu}(t)
$$

whose form follows from Eqs. (2.18), (2.19) within the mean-field approach. At this stage, Eq. (2.22) should be considered together with Eq. (2.14) as a complete system.

\subsubsection{Effect of Autocorrelations in System with One Noise}

Here, we address to a situation where the intensity of one of noises is much larger than others do. The typical picture of the defect structure evolution is shown in Fig. 2 where we present the results of computer simulation of Eqs. (2.18), (2.14) on the grid with $L=100$. The analytic treatment of such a system is achieved on the basis of the unified coloured noise approximation. Formally, if we take the time derivative of Eq. (2.22), replace firstly $\dot{\zeta}$ in terms of $\zeta$ and $\xi$ from Eq. (2.14) and then $\zeta$ in terms of $\dot{x}$ and $x$ from Eq. (2.22), we can obtain the nonMarkovian stochastic differential equation

$$
\tau\left(\ddot{x}-\dot{x}^{2} \frac{\partial}{\partial x} \ln g\right)=-\left[1-\tau\left(f+f_{\text {int }}\right) \frac{\partial}{\partial x} \ln \left(\frac{f+f_{\text {int }}}{g}\right)\right] \dot{x}+f+f_{\text {int }}+g \xi(t)
$$

with $\tau \equiv \tau^{a}$. According to the unified coloured noise approximation $[26,27]$ we use the adiabatic elimination that neglects the terms with $\ddot{x}$ and $\dot{x}^{2}$. Moreover, according to the Ito interpretation of Eq. (2.14), the white noise $\xi(t)$ (last term in Eq. (2.25)) should be considered within the Ito calculus. Hence, we come to the equation

$$
\sigma(x ; \eta) \dot{x}=f(x)+f_{i n t}(x ; \eta)+g(x) \xi(t)
$$

with the kinetic coefficient 

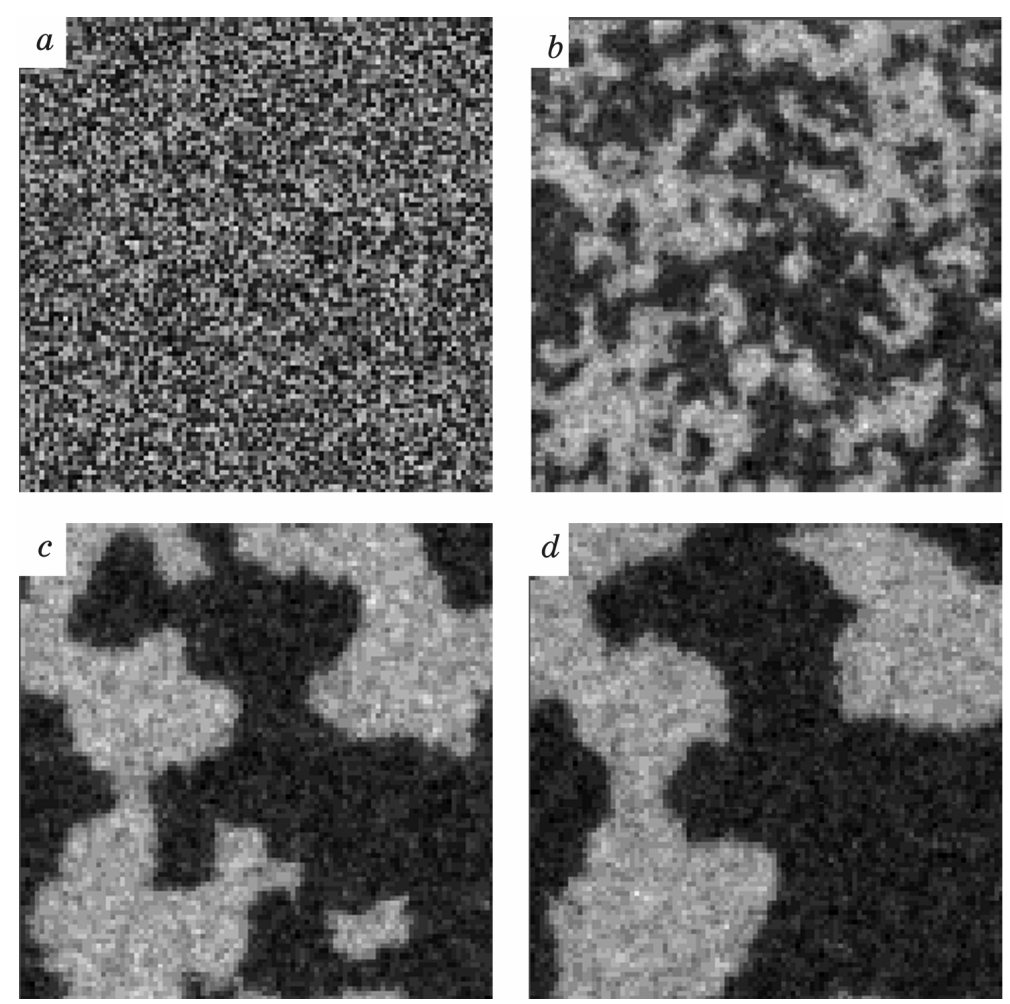

Fig. 2. Time evolution of domains starting in a completely random initial configuration toward an ordered phase for spatially extended model given by Eqs.(2.15), (2.19) for the additive noise on a square lattice $(L=100$, $\left.\theta=\sigma_{x}^{2}=4.0, D=5.0\right)$ at $t=0.0,2.3,10.0,50.4(a, b, c, d)$. Dark areas correspond to a negative values of $x$, light areas to positive values.

$$
\sigma(x ; \eta)=1-\tau\left(f(x)+f_{i n t}(x ; \eta)\right) \frac{\partial}{\partial x} \ln \left(\frac{f(x)+f_{\text {int }}(x ; \eta)}{g(x)}\right) .
$$

Since the stochastic equation (2.24) has continuous set of solutions, one should determine their probability density function $P(x)$ and then calculate the order parameter $\eta=\langle x\rangle$. The evolution equation for $P(x)$ is in the form [28]

$$
\frac{\partial}{\partial t} P(x, t ; \eta)=-\frac{\partial}{\partial x}(\phi(x ; \eta)+\varphi(x ; \eta)) P(x, t ; \eta)+\frac{\sigma^{2}}{2} \frac{\partial^{2}}{\partial x^{2}} \varrho^{2}(x ; \eta) P(x, t ; \eta)
$$

where auxiliary functions are introduced:

$$
\phi(x ; \eta)=\left(f(x)+f_{\text {int }}(x ; \eta)\right) / \sigma(x ; \eta)
$$




$$
\begin{gathered}
\varrho(x ; \eta)=\frac{g(x)}{\sigma(x ; \eta)}, \\
\varphi(x ; \eta)=-\frac{1}{2} \varrho^{2}(x ; \eta) \frac{\partial}{\partial x} \ln \sigma(x ; \eta) .
\end{gathered}
$$

The steady-state solution of Eq. (2.26) has a quasi-Gibbs form

$$
\begin{gathered}
P(x ; \eta)=N^{-1} \exp \left(-U_{e f}(x ; \eta) / \sigma^{2}\right) \\
U_{e f}=-2 \int^{x} \frac{f\left(x^{\prime}\right)+f_{\text {int }}\left(x^{\prime} ; \eta\right)}{g^{2}\left(x^{\prime}\right)} \sigma\left(x^{\prime} ; \eta\right) d x^{\prime}+2 \sigma^{2} \ln g(x)-\sigma^{2} \ln \sigma(x ; \eta)
\end{gathered}
$$

where $N=N(\eta)$ takes care of normalization condition:

$$
N(\eta)=\int_{-\infty}^{\infty} d x \exp \left(-U_{e f}(x ; \eta) / \sigma^{2}\right)
$$

The order-parameter magnitude is defined through the self-consistency condition:

$$
\eta=\int_{-\infty}^{\infty} x P(x ; \eta) d x \equiv F(\eta)
$$

The picture of the noise induced phase transitions is achieved by means of the solutions of the self-consistent Eq. (2.33) and condition

$$
\left.\frac{d F(\eta)}{d \eta}\right|_{\eta=0}=1
$$

to define the phase diagram. For systems where phase transitions with symmetry breaking occur, coefficient Eq. (2.34) has always a root $\eta=0$; nontrivial roots differ only in sign at $d F /\left.d \eta\right|_{\eta=0}>1$ and define the ordered state.

Consider firstly the additive noise of the dislocation density where the multiplicative function is $g_{x}=1$. Within the linear approach we get

$$
\left\langle\dot{x}_{\mathbf{k}}\right\rangle=\left(\theta-1-D|\mathbf{k}|^{2}\right)\left\langle x_{\mathbf{k}}\right\rangle .
$$

Therefore, the solution $\left\langle x_{\mathbf{k}}\right\rangle=0$ becomes unstable at $\theta>\theta_{k}$ where $\theta_{\mathbf{k}}=1+D|\mathbf{k}|^{2}$. It means that the heterogeneity increases the critical magnitude of the control parameter $\theta_{k}$. On the other hand, within the linear approximation the stochastic part does not affect on the stability of the system.

From the phase diagram shown in Fig. 3, it is seen that re- 


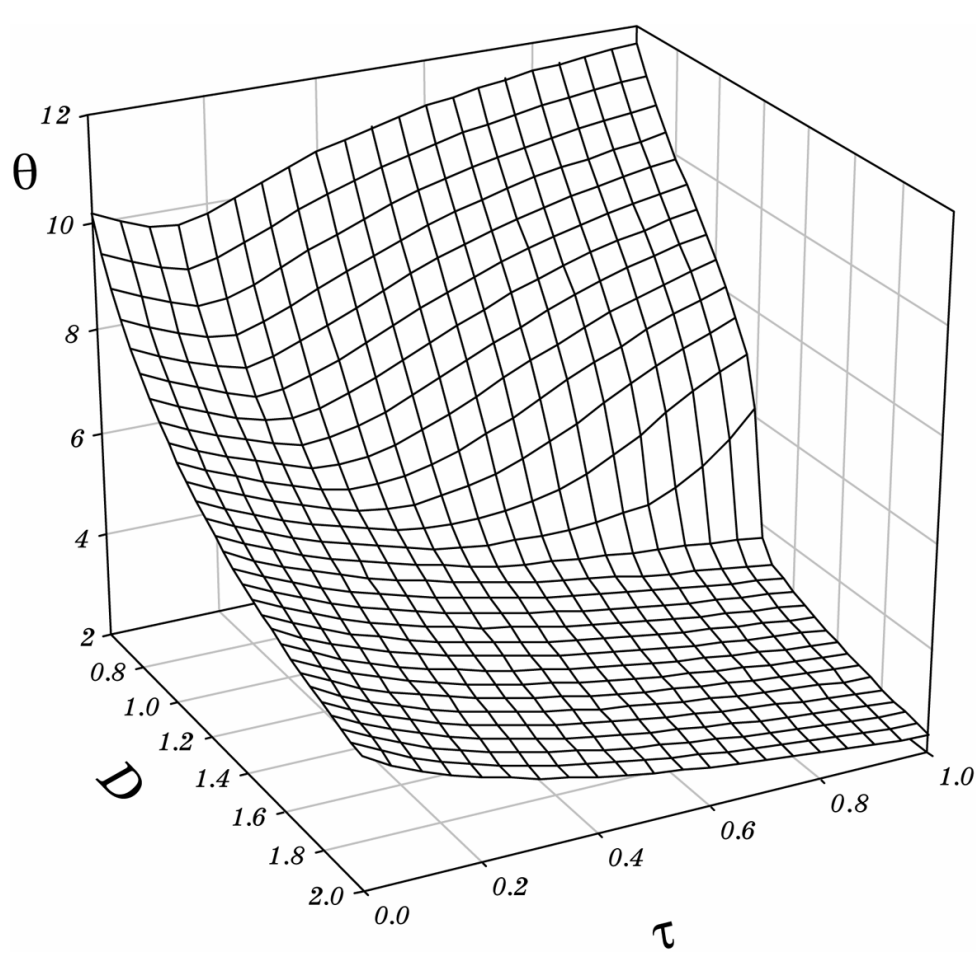

Fig. 3. Phase diagram for the system with additive coloured noise is shown at $\sigma_{x}^{2}=1.0$.

entrant phase transition occurs when we change the spectral characteristic $\tau$ (the domain of the ordered phase is situated above the surface). Here at small $\tau$ we have the chaotic structure of defects ensemble where directions of Burgers vector $b$ are equivalently realized. If we pass through the first threshold $\tau_{1}$, the system becomes as ordered due to the formation of a dislocation cluster with a fixed direction of the Burgers vector. A further increase in $\tau$ (above $\tau_{2}$ ) allows the system to have clusters with two opposite directions of $b$ due to formation of a set of dislocation clusters. Varying the parameter $D$, we can change the size of fluctuation frequency window where the ordered state exists and find the critical magnitudes where one defect structure transforms to another.

Solution of the self-consistency equation (2.33) is shown in Fig. 4, $a$. It is seen that an increase in $\tau$ narrows the domain of the deformation $\theta$ where the ordered state exists. The solutions of Eq. (2.33) are in good correspondence with result of computer simulations in $2 d$ grid (see Figs. $4, b, c$ ).

Let us focus now on the affect of the noise of conjugate field being concentration of point defects with $g_{c}=\left(1+x^{2}\right)^{-1}$. Within the 

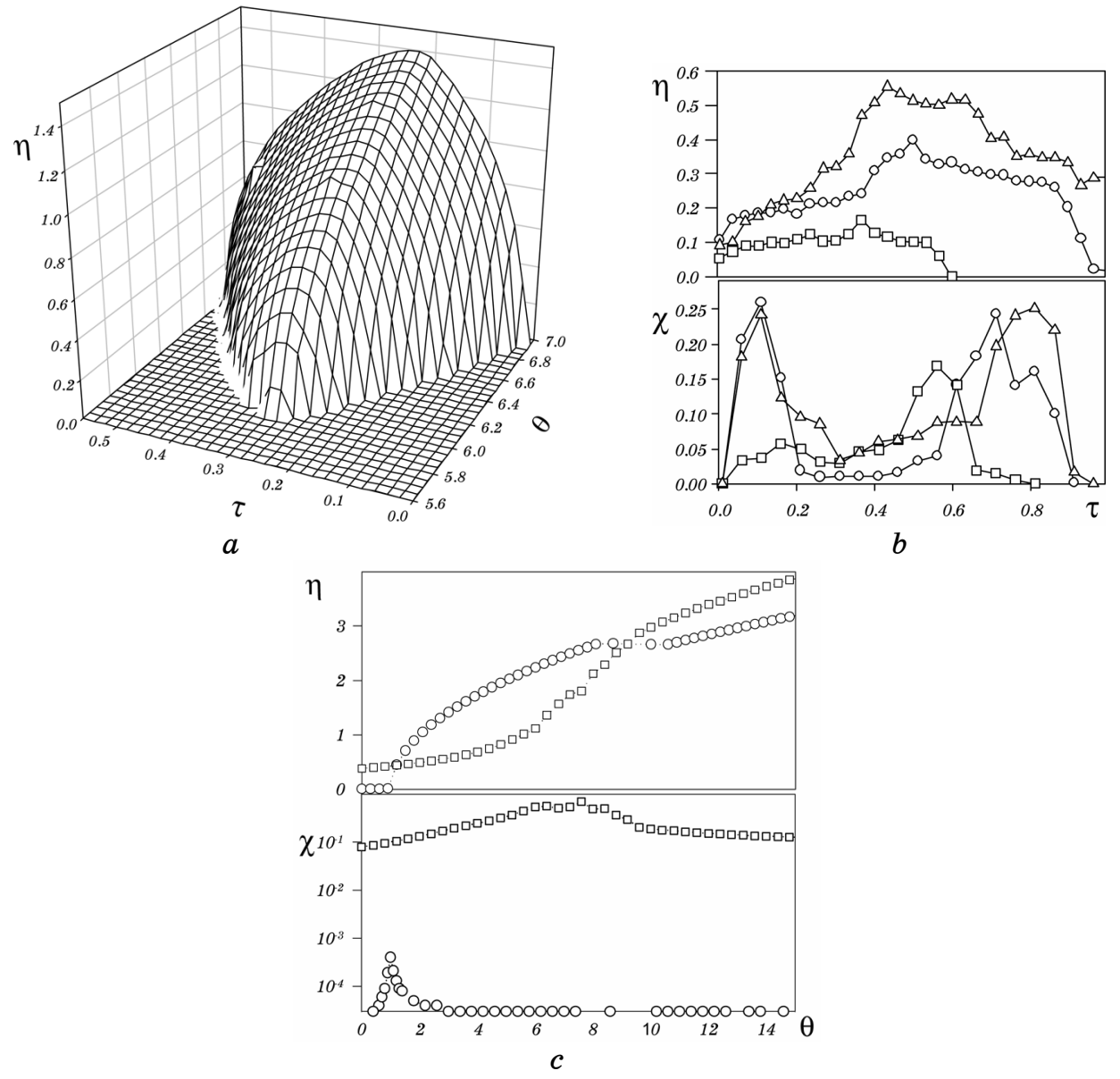

Fig. 4. Order parameter at the additive noise: $a$-dependences $\eta(\theta, \tau)$ is shown at $D=1.0, \sigma_{x}{ }^{2}=9.0 ; b$-order parameter and generalized susceptibility vs. noise autocorrelation time $\tau$ at $\theta=4.0, D=0.7$ (squares); $\theta=4.5$, $D=1.0$ (circles); $\theta=4.0, D=1.0$ (triangles); $c$-order parameter and generalized susceptibility vs. control parameter $\theta$ (circles and squares correspond to $\sigma_{x}{ }^{2}=0.01, D=10.0, \tau=0.1$ and $\sigma_{x}{ }^{2}=49.0, D=10.0, \tau=0.1$ (computer simulations on the grid $32 \times 32$ ).

linear stability analysis, we get renormalization of the critical magnitude for the control parameter through the noise intensity $\sigma_{c}^{2}$. Following the Novikov theorem [29], we obtain

$$
\left\langle\dot{x}_{\mathbf{k}}\right\rangle=\left(\theta-1-2 \sigma_{c}^{2}-D|\mathbf{k}|^{2}\right)\left\langle x_{\mathbf{k}}\right\rangle .
$$

Therefore, the coloured multiplicative noise shifts the transition point to the magnitude of $\theta_{\mathbf{k}}=1+2 \sigma_{c}^{2}+D|\mathbf{k}|^{2}$. 
The corresponding phase diagrams are shown in Fig. 5 where, as in previous case, both the control parameter $\theta$ and noise intensity $\sigma_{c}^{2}$ are varied (domains of ordered and disordered phases are indicated as ' $\mathrm{O}$ ' and ' $D$ '). We should stress that in this case of the multiplicative noise the domain of small values of the noise correlation time $\tau$ is essential. It
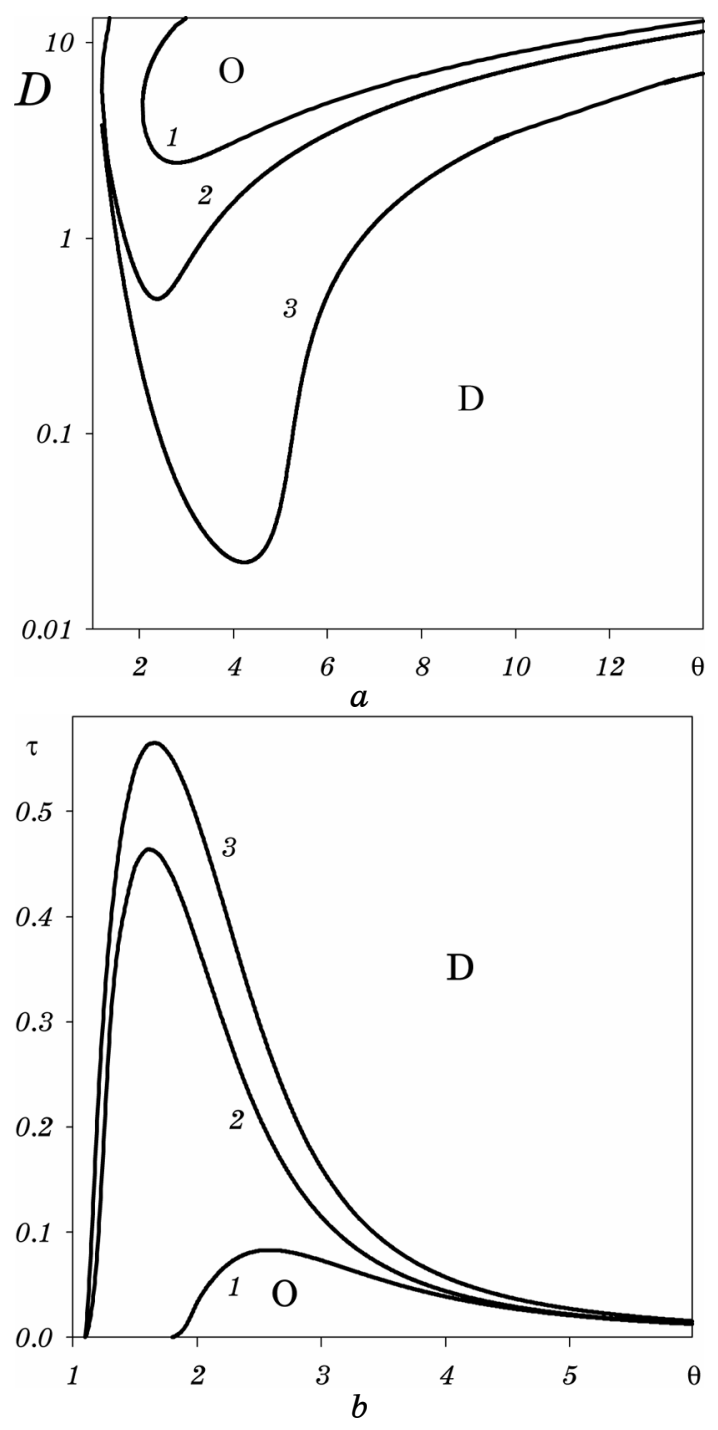

Fig. 5. Phase diagrams for the system with multiplicative colored noise: $a$-dependences $D(\theta)$ are shown at $\tau=0.1, \sigma_{\varepsilon}{ }^{2}=1.18, \tau=0.07, \sigma_{\varepsilon}{ }^{2}=1.0$; $\tau=0.01, \sigma_{\varepsilon}{ }^{2}=1.0 ;$ (curves $1,2,3$ ); $b$-dependences $\tau(\theta)$ are shown at $D=0.9, \sigma_{\varepsilon}{ }^{2}=1.18, D=0.9,{\sigma_{\varepsilon}}^{2}=1.0, D=1.2, \sigma_{\varepsilon}{ }^{2}=0$ (curves $\left.1,2,3\right)$. 
is seen from Fig. 5, $a$ the ordered phase is realized within bounded domain of control parameter and diffusion constant. At extremely small $\tau$, there is only one transition along $D$ axis (curve 3), whereas a slight increase in $\tau$ shrinks the region of ordered phase (curve 1,2). According to Fig. 5, $b$, the system with multiplicative noise is more sensitive to the variation of the noise autocorrelation time. Here, with an increase in $\tau$ the ordered state $\eta \neq 0$ is realized in the shrinking domain of values of the deformation $\theta$. An increase in $D$ expands the domains of noise correlation values and increases the size of the control parameter values where the ordering occurs. Notice that in the case of multiplicative noise only one transition occurs along $\tau$ axis, whereas in the case of additive noise the system undergoes two transitions (re-entrant ordering transition).

Corresponding behaviour of the order parameter is shown in Fig. 6 where both analytical and numerical results are presented in Figs. 6, $a$, $b$ ). Here, an increase in the deformation $\theta$ results in a rebuilding the defect structure. Further, an increase in $\theta$ suppresses the process of the dislocation cluster formation with unique direction of the Burgers vector.

The noise of the control parameter changes crucially the system behaviour. Indeed, related amplitude $g_{s}$ becomes trivial at $x=0$ which corresponds to formation of the absorbing state [5, 30]. Here the stationary solution of the Fokker-Planck equation becomes unnormalizable [31] to mean the freezing the configuration points of the phase space when the domain $x<<1$ is reached [32,33]. Therefore, in course of time, the density of dislocations attains the value corresponded to chaotic structure, and noise does not allow preceding an ordering process. Indeed, in the limit $x \rightarrow 0$ the distribution function is characterized by the power law construction

$$
P \propto x^{-2(1+D-\theta) / \sigma^{2}-3}, \quad x \rightarrow 0 .
$$

Due to $D>0$, the logarithm singularity in Eq. (2.37) can be avoided only at $\theta>D+1+\sigma^{2}$.

\subsubsection{System with Two Noises}

Considering a stochastic system with more than one noise, we have to deal with an effect of correlation between them. As shown in Ref. [34], the system with two noises behaves itself further in unusual manner. One holds the opinion that cross correlation introduces only weak corrections to results obtained for uncorrelated fluctuations.

Here, we demonstrate the crucial role of cross correlation between fluctuations and show that they can change the order of phase transitions. Because such a problem can not be solved correctly by using the 

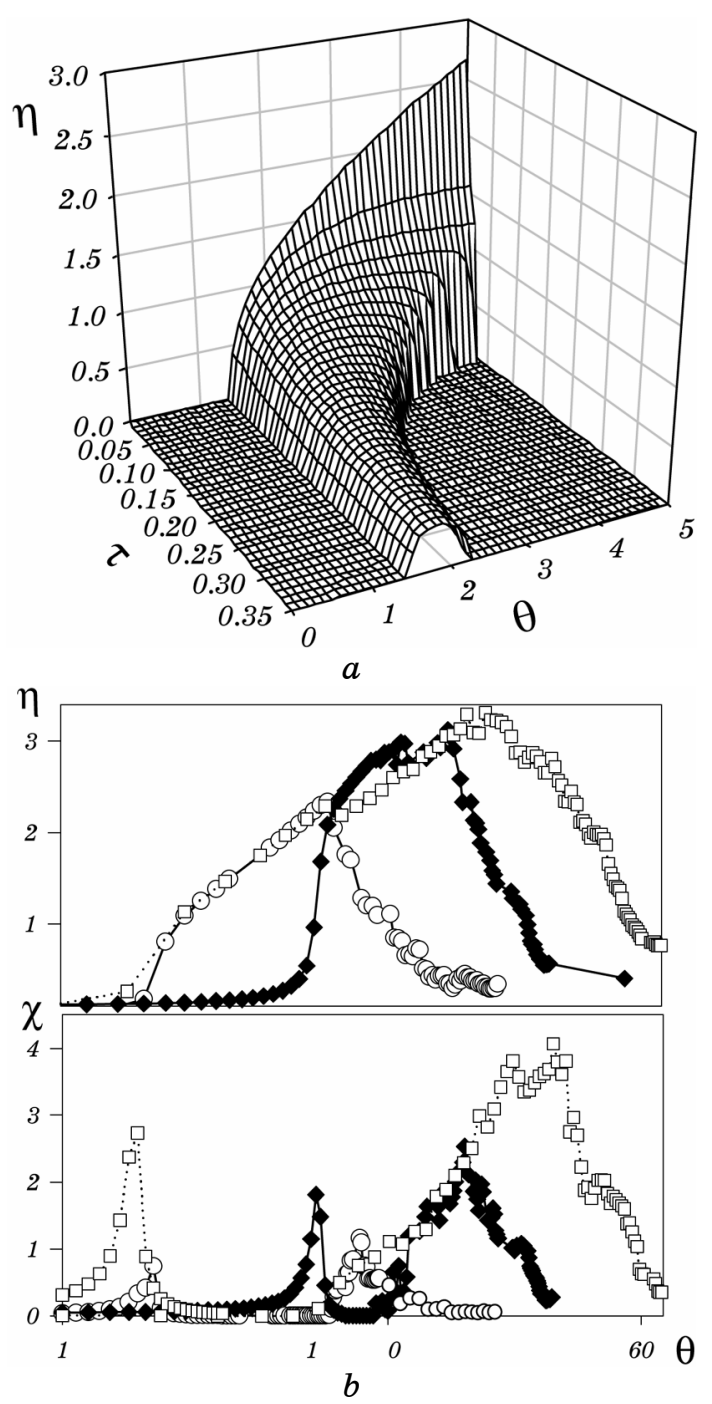

Fig. 6. Order parameter at the multiplicative noise: $a$-dependences $\eta(\theta, \tau)$ are shown at $D=1.0, \sigma_{\varepsilon}{ }^{2}=1.0 ; b$-order parameter and generalized susceptibility vs. deformation $\theta$ (diamonds, circles and squares correspond to $\tau=0.1, \quad D=2.0, \quad \sigma_{\varepsilon}{ }^{2}=25 ; \quad \tau=0.1, \quad D=2.0, \quad \sigma_{\varepsilon}{ }^{2}=1.0 ; \quad \tau=0.1, \quad D=8.0$, $\sigma_{\varepsilon}{ }^{2}=1.0$ (computer simulations on the grid $32 \times 32$ are shown at the same parameters as analytic dependences).

standard methods based on the Novikov theorem [29] or the unified coloured noise approximation [26, 27], we apply the cumulant expansion method proposed by Van Kampen [35] and developed in Ref. [36].

To construct an equation for the probability density $\rho\left(\left\{x_{k}\right\}, t\right)$ we 
exploit a conventional device and proceed from the continuity equation

$$
\frac{\partial}{\partial t} \rho\left(\left\{x_{k}\right\}, t\right)=-\sum_{k} \frac{\partial}{\partial x_{k}}\left(\dot{x}_{k} \rho\left(\left\{x_{k}\right\}, t\right)\right) \text {. }
$$

The probability density function is given by the averaging over noise, i.e. $P\left(\left\{x_{k}\right\}, t\right)=\left\langle\rho\left(\left\{x_{k}\right\}, t\right)\right\rangle$. Inserting the time derivative from Eq. (2.16) into Eq. (2.38), we have

$$
\frac{\partial}{\partial t} \rho\left(\left\{x_{k}\right\}, t\right)=-\sum_{k}\left[L_{k}-\frac{\partial}{\partial x_{k}} \sum_{\mu} g_{\mu k} \xi_{\mu k}\right] \rho\left(\left\{x_{k}\right\}, t\right),
$$

where the evolution operator is denoted as

$$
L_{k}=\frac{\partial}{\partial x_{k}}\left(f_{k}+\frac{D}{2 d} \sum_{j} D_{k j} x_{j}\right) .
$$

According to the well-known cumulant expansion method [35] noise averaging of Eq. (2.39) derives to the following expression for the probability density $P\left(\left\{x_{k}\right\}, t\right)[34]$

$$
\frac{\partial}{\partial t} P=\sum_{k}\left[-L_{k}+\sum_{\mu, v} L_{\mu k}^{(0)} \int_{0}^{\infty} C_{\mu v}(t, t-\tau) e^{-L_{k} t} L^{(0)}{ }_{v k} e^{L_{k} t} d \tau\right] P
$$

where we put $P \equiv P\left(\left\{x_{k}\right\}, t\right)$,

$$
L_{\mu i}^{(0)} \equiv \frac{\partial}{\partial x_{k}} g_{\mu k}=\frac{\partial}{\partial x_{k}} g_{\mu}\left(x_{k}\right),
$$

and assume the physical time to be much more some correlation scale $\tau_{\mu, v}\left(t>>\tau_{\mu, v}, \tau_{\mu, \mu}\right)$. Then, expansion of exponents arrives at the perturbation expansion

$$
\frac{\partial}{\partial t} P=\sum_{k}\left[-\frac{\partial}{\partial x_{k}}\left(f_{k}+\frac{D}{2 d} \sum_{j} D_{k j} x_{j}\right)+\sum_{n=0}^{\infty} \sum_{\mu, v} L_{\mu k}^{(0)} C_{\mu \nu}^{(n)} L_{\mu k}^{(n)}\right] P
$$

where moments of the correlation function are determined as

$$
C_{\mu \nu}^{(n)}=\frac{1}{n !} \int_{0}^{\infty} \tau^{n} C_{\mu v}(t, t-\tau) d \tau .
$$

Respectively, operators $L_{v}^{(n)}$ are defined through the commutator

$$
L_{\mu i}^{(n)}=\left[L_{\mu k}^{(n-1)}, L_{k}\right] \text {. }
$$

So, the first-order approximation yields 


$$
L_{\mu k}^{(1)}=\frac{\partial}{\partial x_{k}} g_{\mu k} \frac{\partial}{\partial x_{k}}\left(f_{k}+\frac{D}{2 d} \sum_{j} D_{k j} x_{j}\right)-\frac{\partial}{\partial x_{k}}\left(f_{k}+\frac{D}{2 d} \sum_{j} D_{k j} x_{j}\right) \frac{\partial}{\partial x_{k}} g_{\mu k}
$$

According to obtained expressions, the zero-order contribution of correlations gives the following terms for the Kramers-Moyal expansion [37]

$$
\begin{aligned}
& D_{1}^{(0)}=\sum_{\mu, v} C_{\mu \nu}^{(0)} g_{\mu k} \frac{\partial g_{v k}}{\partial x_{k}}, \\
& D_{2}^{(0)}=\sum_{\mu, v} C_{\mu \nu}^{(0)} g_{\mu k} g_{v k} ;
\end{aligned}
$$

accordingly, a contribution of first-order terms gives

$$
\begin{aligned}
& D_{1}^{(1)}=\sum_{\mu, v} C_{\mu \nu}^{(1)} \frac{\partial g_{v k}}{\partial x_{k}}\left[\left(f_{k}+\frac{D}{2 d} \sum_{j} D_{k j} x_{j}\right) \frac{\partial g_{\mu k}}{\partial x_{k}}+g_{\mu k} \frac{\partial}{\partial x_{k}}\left(f_{k}+\frac{D}{2 d} \sum_{j} D_{k j} x_{j}\right)\right], \\
& D_{2}^{(1)}=\sum_{\mu, v} C_{\mu \nu}^{(1)} g_{v k}\left[\left(f_{k}+\frac{D}{2 d} \sum_{j} D_{k j} x_{j}\right) \frac{\partial g_{\mu k}}{\partial x_{k}}+g_{\mu k} \frac{\partial}{\partial x_{k}}\left(f_{k}+\frac{D}{2 d} \sum_{j} D_{k j} x_{j}\right)\right] .
\end{aligned}
$$

Therefore, the effective Fokker-Planck equation reads

$$
\frac{\partial}{\partial t} P=\sum_{k} \frac{\partial}{\partial x_{k}}\left[-D_{1}\left(x_{k}\right)+\frac{\partial}{\partial x_{k}} D_{2}\left(x_{k}\right)\right] P,
$$

here the drift and the diffusion coefficients are given by

$$
\begin{aligned}
& D_{1}\left(x_{k}\right)=f_{k}+\frac{D}{2 d} \sum_{j} D_{k j} x_{j}+\sum_{n=0} D_{1}^{(n)}\left(x_{k}\right), \\
& D_{2}\left(x_{k}\right)=\sum_{n=0} D_{2}^{(n)}\left(x_{k}\right) .
\end{aligned}
$$

Integrating Eq. (2.49) over all variables, with the exception of $x_{k}$, and using the fact that the steady state properties are isotropic and translationally invariant, one obtains the following stationary equation for the one-site probability:

$$
\left[-D_{1}\left(x_{k}\right)+\frac{\partial}{\partial x_{k}} D_{2}\left(x_{k}\right)\right] P\left(x_{k}\right)=0
$$

where conditions $P\left(x_{i}\right)=0, \partial P / \partial x_{i}=0$ at $x_{i} \rightarrow \infty$ are taken into account. Within the mean-field theory, the interaction term is in the form $\frac{D}{2 d} \sum_{j} D_{k j} x_{j}=D\left(\eta-x_{k}\right)$ where the $\eta$ value is defined from the selfconsistency equation (2.33). Then, equation (2.51) gives the stationary distribution function 


$$
P(x, \eta)=N^{-1}(\eta) D_{2}^{-1}(x, \eta) \exp \left(\int_{0}^{x} \frac{D_{1}\left(x^{\prime}, \eta\right)}{D_{2}\left(x^{\prime}, \eta\right)} d x^{\prime}\right) .
$$

Considering the uncorrelated noises, we start with expression $C_{x, \varepsilon}\left(t, t^{\prime}\right)=\sigma_{x} \sigma_{\varepsilon} \delta\left(t-t^{\prime}\right)$. In order to suppress correlations defined through the Novikov theorem [29], we put $C_{x, \varepsilon}^{(0)}=0$ that derives to the standard Fokker-Planck equation with independent noises. Thereby, the terms appearing in Eq. (2.49) can be calculated to be

$$
\begin{aligned}
D_{1}^{(0)} & =\sigma_{\varepsilon}^{2} g_{\varepsilon} \frac{\partial g_{\varepsilon}}{\partial x}, \\
D_{1}^{(1)} & =\sigma_{\varepsilon}^{2} \tau \frac{\partial g_{\varepsilon}}{\partial x} \frac{\partial}{\partial x} g_{\varepsilon}\left(f+f_{i n t}\right), \\
D_{2}^{(0)} & =\sigma_{x}^{2}+\sigma_{\varepsilon}^{2} g_{\varepsilon}^{2}, \\
D_{2}^{(1)} & =\sigma_{\varepsilon}^{2} \tau g_{\varepsilon} \frac{\partial}{\partial x} g_{\varepsilon}\left(f+f_{i n t}\right) .
\end{aligned}
$$

Inserting $D_{1}, D_{2}$ into Eq. (2.52) and the obtained result into Eq. (2.34), we get an equation to construct the phase diagram. A related solution is shown in Fig. 7 where letters ' $O$ ' and ' $\mathrm{D}$ ' denote the domains of ordered and disordered phases respectively. Note that the selfconsistency equation has two nontrivial solutions, which are differing in sign. Hence, following the standard scheme we classify this transition as phase transition with the symmetry breaking. The ordered

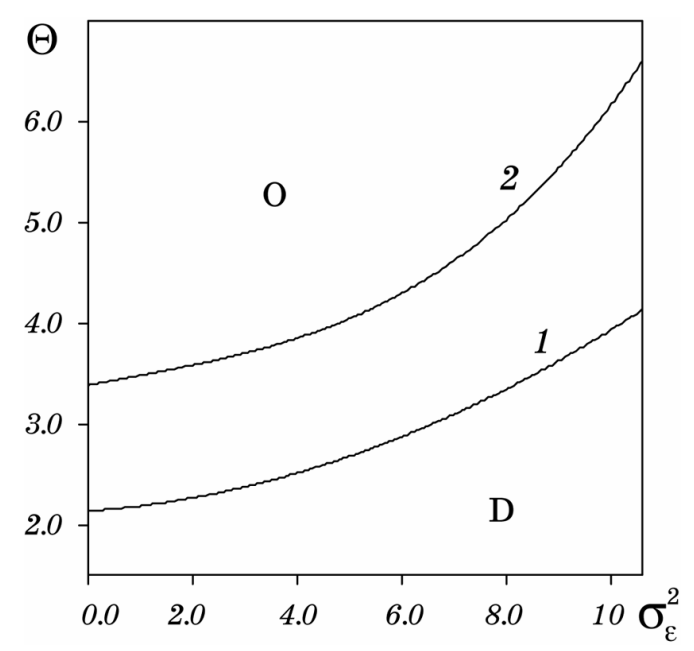

Fig. 7. Phase diagram at $\sigma_{x}^{2}=1.0, \tau=0.01$ : curves 1,2 correspond to $D=0.9, D=0.5$. 


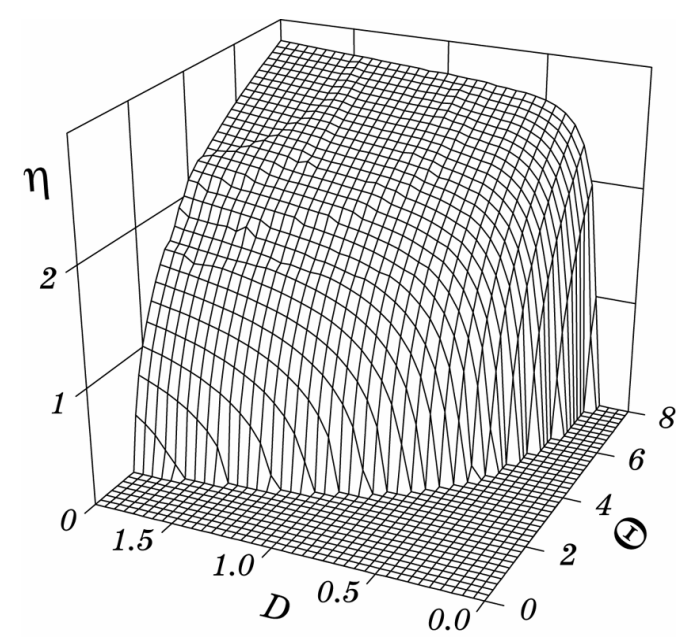

Fig. 8. Order parameter $\eta$ vs. the control parameter $\theta$ and the coupling constant $D$ at $\sigma_{x}=\sigma \varepsilon=1.0, \tau=0.01$.

phase is seen to be realized at large values of the control parameter $\theta$, so that the noise induces the transition of a second order from the ordered phase to disordered one. Another conclusion is that the system is ordered at large values of the coupling constant $D$. Thus, we arrive at the standard synergetic picture of phase transition where the system becomes ordered one when the control parameter increases. As shown in Fig. 8, the order parameter $\eta$ increases monotonically beyond the critical magnitudes of $\theta$ and $D$.

Most interesting picture of noise induced phase transition can be found when the noises are correlated. Taking into account terms in Eqs. (2.47), (2.48) with $\tau^{c} \equiv \tau_{\varepsilon x}^{c} \neq 0$, we obtain

$$
\begin{gathered}
D_{1}^{(0)}=\sigma_{\varepsilon}\left(\sigma_{x}+\sigma_{\varepsilon} g_{\varepsilon}\right) \frac{\partial g_{\varepsilon}}{\partial x}, \\
D_{1}^{(1)}=\sigma_{\varepsilon} \frac{\partial g_{\varepsilon}}{\partial x} \frac{\partial}{\partial x}\left[\left(\tau_{c} \sigma_{x}+\tau \sigma_{\varepsilon} g_{\varepsilon}\right)\left(f+f_{i n t}\right)\right], \\
D_{2}^{(0)}=\sigma_{\varepsilon}^{2}\left[\sigma_{x} \sigma_{\varepsilon}^{-1}\left(\sigma_{x} \sigma_{\varepsilon}^{-1}+2 g_{\varepsilon}\right)+g_{\varepsilon}^{2}\right], \\
D_{2}^{(1)}=\sigma_{\varepsilon}^{2}\left[\sigma_{x} \sigma_{\varepsilon}^{-1} \tau^{c}\left(\frac{\partial}{\partial x} g_{\varepsilon}+g_{\varepsilon} \frac{\partial}{\partial x}\right)\left(f+f_{\text {int }}\right)+\tau g_{\varepsilon} \frac{\partial}{\partial x} g_{\varepsilon}\left(f+f_{\text {int }}\right)\right] .
\end{gathered}
$$

In accordance with the self-consistency equation, the order parameter $\eta$ varies depending on the control parameter $\theta$ as is plotted in Fig. 9. Such a behaviour is determined by the principle contribution into Eq. (2.33) given by the zero-order term $C_{x, \varepsilon}^{(0)}$. It contains the construction to break the symmetry of probability-density function (2.53) even in the 
case of $D=0$, and therefore we always have solution of Eq. (2.33) such as $\eta \neq 0$. Next, we define such an ordered state as a matrix phase.

Let the additive noise intensity be fixed, whereas the intensity of the multiplicative noise of the control parameter takes on increasing values. Then, at small $\sigma_{\varepsilon}$ (Fig. 9, $a$ ) the order parameter takes a nontrivial positive magnitude at small values of the control parameter where the ordered phase is stable. A further increase in $\theta$ brings the system to the state with negative solution of the self-consistency equation. Physically it means a reorientation transition at $\theta=\theta_{r}$ where the average Burgers vector of dislocation clusters change the direction. This solution is unique until the control parameter reaches the critical value $\theta_{c}$ where the bifurcation occurs. Then, two positive solutions of
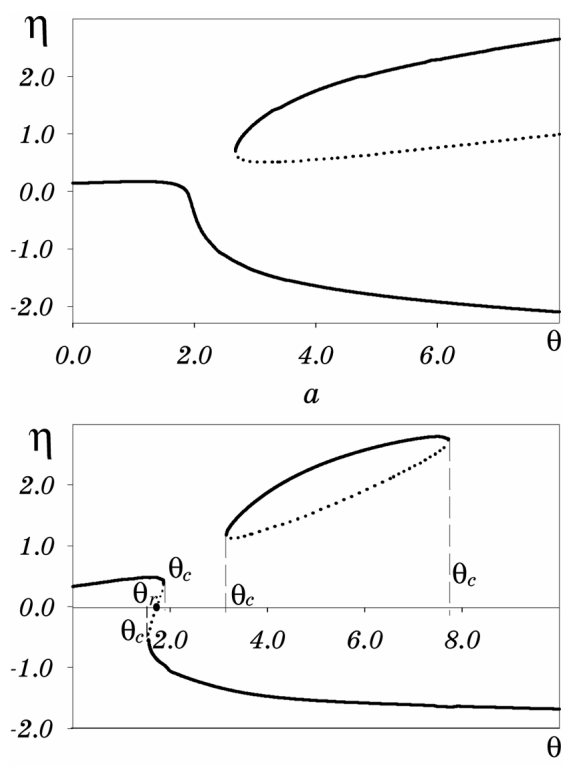

$b$

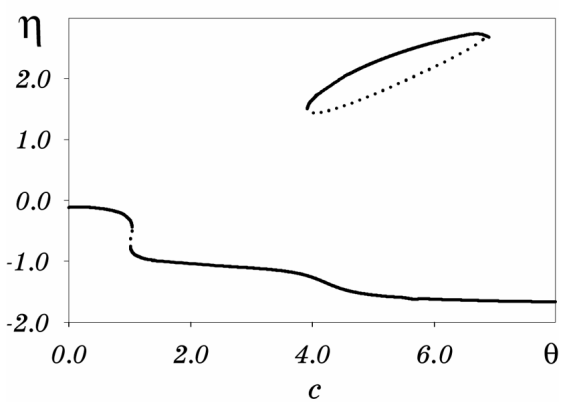

Fig. 9. Order parameter vs. the control parameter at $\sigma_{x}=1.0, \tau=\tau_{c}=\mathbf{0 . 0 1}$, $D=0.9: a-\sigma_{\varepsilon}^{2}=0.09 ; b-\sigma_{\varepsilon}^{2}=1.0 ; c-\sigma_{\varepsilon}^{2}=1.69$. 
Eq. (2.33) appear which correspond to a metastable state formation. In such a case, we conclude that the positive solution at small $\theta$ and negative one at large $\theta$ means the matrix phase. A new phase formation can be addressed to branches of the metastable state.

Following the standard theory, we can classify the appearance of new phase as a biased phase transition. According to Fig. 9, $b$, the reentrance of the new phase formation is observed when we increase the intensity of the multiplicative noise. Here, the new phase is realized at $\theta \in\left[\theta_{c}, \theta^{c}\right]$. In the domain of small $\theta$, the hysteresis loop is observed, and the order parameter takes the negative magnitude in discontinuous manner. Such a metastable state corresponds to a situation where the Burgers vector of dislocation clusters can have two opposite directions and some amount of clusters with fixed direction of $b$ can be metastable. Besides, the new phase at large $\theta$ is formed due to the bifurcation. Both metastable phases appeared in such phase transitions have different mechanisms of their formation. In such a case, above-mentioned phases can be associated with different defect structures appearing in the course of plastic deformation. Finally, a further increase in the noise
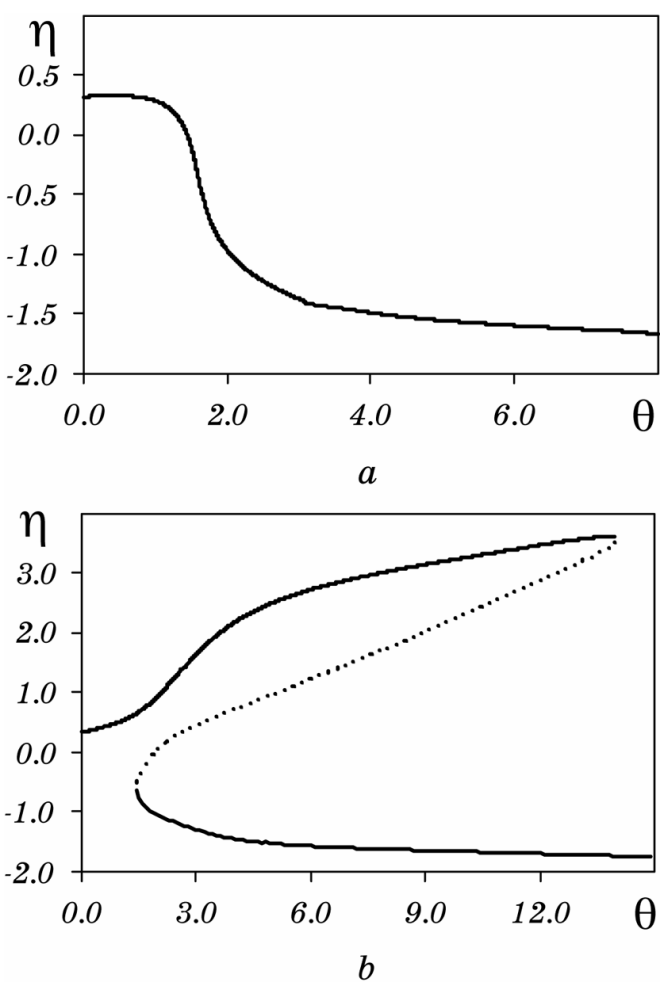

Fig. 10. Order parameter vs. the control parameter at $\sigma_{x}=\sigma_{\varepsilon}=1.0, \tau=\tau_{c}$ : $a-$ $D=0.5 ; b-D=1.2$. 
intensity leads to the disappearing hysteresis loop at small $\theta$, and the matrix phase is characterized by the negative magnitude of $\eta$.

The influence of the spatial coupling constant $D$ on the picture of the system behaviour is shown in Fig. 10. It is seen, that at small $D$ (Fig. 10, a), we have unique solution of the self-consistency equation which defines the matrix phase. Here the order parameter changes the sign when we increase the control parameter. At intermediate $D$, the new phase appears due to bifurcation and at large $D$ the branches of the new and matrix phases are merged. It results to formation the hysteresis loop on which the order parameter changes the sign. The related solution for the phase diagram arrives at the picture shown in Fig. 11 where the control parameter $\theta$ is built as the function of the multiplicative noise intensity $\sigma_{\varepsilon}^{2}$ at different values of the coupling constant $D$ and the noise cross correlation time $\tau_{c}$. We denote the domain of the matrix phase as ' $\mathrm{P}$ ' and ' $\mathrm{N}$ ' to indicate the positive and negative magnitudes of the order parameter; the new phase we denote as ' $N$ '. The appearance of the domain of the new phase allows to classify such transitions as first order phase transitions. The main peculiarities of the system's behaviour are as follows: (i) an increase in the cross correlation time $\tau_{c}$ is accompanied by extending the domains of a metastable state (cf. Figs. 11, $a, b)$; (ii) an increase in the spatial coupling constant $D$ is accompanied by the increase of the domain of a new phase ('N') (cf. Figs. 11, c).

The performed analysis shows the crucial role of fluctuations. Considering the one coloured noise in Section 2.2.2, we address the situation where reconstruction of defect structure is observed. Accounting the correlation between noises in Section 2.2.3 allows us to consider such intermediate structures whose formation is shown as a chain of phase transitions during the process of plastic deformation.

\section{REGIME OF SELF-ORGANIZED CRITICALITY}

Among the equilibrium phase transitions or noise-induced ones, a special regime of the system behaviour is known as a self-organized criticality (SOC). It explains spontaneous (avalanche-type) dynamics, unlike the typical phase transitions that occur only when a control parameter is driven above a critical value. A main feature of the systems displaying SOC is their self-similarity that leads to a power-law distribution over avalanche sizes. The SOC behaviour appears in a vast variety of systems, such as real sand pile (ensemble of grains of sand moving along increasingly tilted surface) [38-41], intermittency in biological evolution [42], earthquakes and forest-fires, depinning transitions in random medium and explosive crystallization [43].

In this Section, we present an analytical approach, which is able to describe the behaviour of a whole avalanche ensemble in a phenomenological manner considering the process of explosive crystallization. 

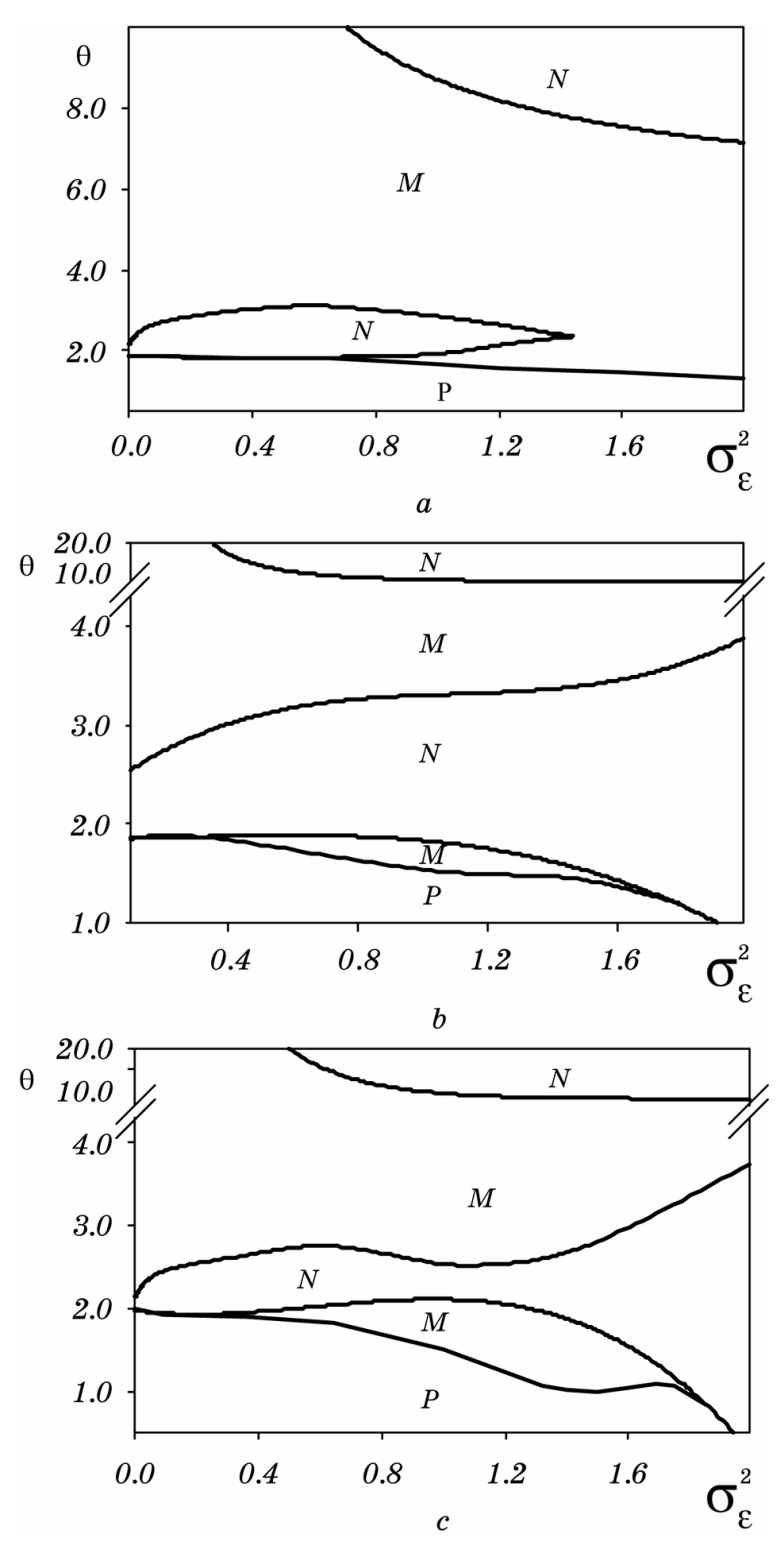

Fig. 11. Phase diagram at $\sigma_{x}=1.0, \tau_{c}=0.01: a-D=0.9, \tau_{c}=0.05 ; b-$ $D=0.9, \tau_{c}=0.01 ; c-D=0.95, \tau_{c}=0.01$.

In Section 3.1, we use that approach to describe the explosive crystallization process, which manifest the SOC regime. In Section 3.1.1, the experimental data is presented to show that a course of explosive crystallization of ultradisperse amorphous materials is determined by high density of crystalline phase embryos. The crystallization phase spread- 
ing is similar to percolation cluster formation under consideration of liquid flow in a random medium [44]. Section 3.1.2 is devoted to investigations of conditions of this picture as a result of self-organization is carried out. This process is fixed by the velocity of crystallization front motion, its temperature, and difference of the thermodynamic potentials of amorphous and crystalline states. In Section 3.2, the direct examination of explosive crystallization is carried out as a self-organized criticality process caused by fractional feedback and stochastic influence of the main modes of the system. This assumption allows us to describe the subcritical regime of the avalanche formation in natural manner. The above generalization puts forward basis of Section 3.2.1 devoted to consideration of avalanche ensemble. Following famous Edwards paradigm $[13,14]$, an effective scheme addressed to nonextensive thermodynamics [15] is proposed to determine a time-dependent distribution over energies of equipped sites in the process of crystallization. In Section 3.2.2, we show that this distribution is the solution of both nonlinear Fokker-Planck equation that appears in the description of nonextensive systems [15], and fractional Fokker-Planck equation inherent in Lévy-type processes characterized by a dynamical exponent $z$ [16]. As a result, we obtain relations between the exponent $\tau$ of the distribution inherent to the SOC regime, fractal dimension $D$ of phase space, characteristic exponent of multiplicative noise, a number of governing equations needed to present self-consistent behaviour in SOC regime, dynamical exponent $z$, and Tsallis nonextensivity parameter $q$.

\subsection{Formation of Avalanche in the Explosive Crystallization Process}

The metastable amorphous films are obtained usually by quenching of a melt or by steam condensation on a cold substrate [45-46]. Experiments show a vast variety and complicated character of the subsequent transitions into the stable crystalline state. If the film thickness is so small that the crystallization heat can be absorbed by a thermostat, the ordinary cold crystallization mechanism works [12]. So, in the crystallized films of semiconductors, the undulating surface is developed under the formation of combs, whose long axes are perpendicular to the direction of the crystallization front movement [47]. As is known, at small number of the crystal embryos, this mechanism is realized if both the nucleation frequency of crystallization centres $J$ and the velocity of crystal growth $u$ are very small.

Sometimes, emerging explosive crystallization can be initiated by the local heating (for example, by laser or electron impulse). Such a scenario takes place in the case of instability appearance of the interfacial boundary motion due to both the heat exchange with substrate and the influence of laser radiation [47]. This instability is ensured with a non-linear dependence of the crystal growth velocity $u$ as a function of tempera- 
ture. Moreover, the crystallization front instability can be fluctuating in character that is manifested in the experiments with undulation of crystallized surface [43]. Such behaviour appears in the partially crystallized film or at the incomplete crystallization of amorphous phase.

Another scenario is observed in the amorphous medium in which as the crystal growth velocity $u$, so the nucleation rate $J$ of embryonic crystals are sufficient large. Indeed, at low temperatures, the quantities $J$ and $u$ increase with temperature growth, so that the self-heating stimulates the crystallization. Therefore, the increase of a film thickness can lead to a situation, when the crystallization heat cannot be absorbed by an environment that causes the heat instability [48]. As a result, the spontaneous transition to regime of the explosive crystallization can be provided by the heating effect. Examples of such amorphous mediums are the amorphous ice layers, the some organic matters $[12,45]$, and the layers of the germanium amorphous ultradisperse powder with the admixture of the crystalline phase $[45,46]$.

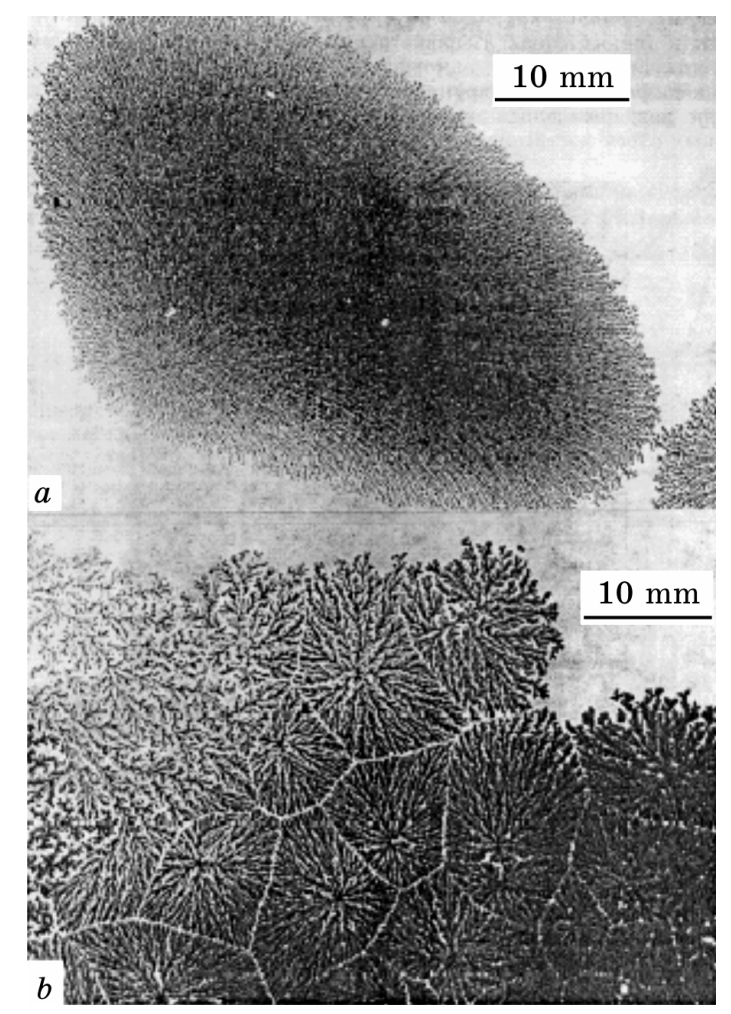

Fig. 12. The patterns which arise in the layer of amorphous powder of Ge at the explosive crystallization: $(a)$ from single center; $(b)$ from several centers which arise spontaneously. 


\subsubsection{Experimental}

The experimental study of influence of the crystal inclusions distributed in the amorphous phase volume in the kinetics of the explosive crystallization is carried out with the germanium [46]. Unfortunately, there is no information in literature about spontaneous rise of the explosive crystallization in continuous amorphous thin films of germanium. It is known only that the maximum value of formation frequency of crystalline embryos is $J_{\max } \sim 10^{14} \mathrm{~s}^{-1} \mathrm{~cm}^{-3}$ in the supercooled germanium and corresponds to the more high temperatures than realized at the explosive crystallization process [12]. Therefore, in the germanium amorphous films the natural process of crystals nucleation has not enough intensity for significant influence in the explosive crystal growth. Let us point out in this connection that in amorphous ice layers, where the spontaneous explosive crystallization takes place, one has $J_{\max } \sim 10^{20} \mathrm{~s}^{-1} \mathrm{~cm}^{-3}$.

In order to intensify the mentioned influence of crystal inclusions, the experiments were carried out with the thin films of amorphous nanopowders with admixture of large number of smallest crystals having not more $3-10 \%$ of total mass. The layers of amorphous powder with the characteristic size of particles $3-10 \mathrm{~nm}$ were obtained by thermal evaporation and following condensation of germanium in the atmosphere of inert gas at the pressures 10-100 Pa. Changing the evaporation intensity allows us to regulate the part of the crystalline particles in amorphous powder. Another peculiarity of our experiments is that the substrates absorb significantly smaller heat due to porosity of amorphous films.

The spontaneous explosive crystallization has been observed in layers of nanopowders with the thickness $0.01-0.1 \mathrm{~mm}$ at the substrate temperature $300-400 \mathrm{~K}$. In dependence on initial concentration of crystalline phase, the movement velocities of crystallization wave have been changed in the range $0.01-0.1 \mathrm{~m} / \mathrm{s}$. Contrary to the transverse undulation being inherent in the usual crystallization mechanism, in our case the front movement leads almost always to formation of 'twigs' along the movement direction. The characteristic pictures of explosive crystallization in the powder layers are shown in Fig. 12, where the light background corresponds to non-crystallized domains. It is seen that the rise of explosive crystallization avalanches occurs from the single centres, which act as original embryos. The cornerstone of our observation is that the crystalline phase distribution has a fractal character being similar to the pattern appeared in diffusion limited aggregation [8].

\subsubsection{Conditions to the Explosive Crystallization Process}

The experimental data show that two mechanism of amorphous mate- 
rial crystallization, depending on external conditions and presence of crystalline phase embryos, are possible: the slow growth of a cold crystal and the explosive crystallization that is caused by the phase transformation heating. According to [46] the transition between these regimes is jump-like in character, as a first-order phase transition. We will show below that such a transition is caused by the system selforganization due to the positive feedback between the heating and growth velocity of crystalline phase.

To analyze the problem, the synergetic approach is used where the time dependences of the crystallization front velocity $u(t)$, its temperature $T(t)$, and the specific crystallization heat $\phi$ (thermodynamic potential, which can play a role of entropy as a conjugate parameter to the temperature). The equations define these dependences to take into account their dissipative character and the positive feedback between quantities $u$ and $\phi$ that is the reason for self-organization. On the other hand, in order to provide the stability of a system we introduce also the negative feedback between $u$ and $T$. In the framework of proposed considerations, the evolution equations for $u, T$ and $\phi$ should coincide formally with the Lorenz system (see Eq. (2.2)) that is the simplest way to describe the self-organization process [2]. Here instead of $\varepsilon, d$ and $\tau$ we use the crystallization front velocity $u$, temperature $T$ and potential $\phi$. In the adiabatic limit of the noiseless systems where the relaxation time for the order parameter (being the velocity $u$ ) $t_{u}$ is larger then relaxation times $t_{T}, t_{\phi}$ for the conjugate field $T$ and control parameter $\phi$, the Lorenz system describes the second-order transition. However, the cold crystallization mode transforms into the explosive one in accordance with the first-order mechanism. To avoid this discrepancy, the simplest approximation was used [43]

$$
\frac{1}{t_{u}}=\frac{1}{t_{0}}\left(1+\frac{\kappa}{1+\left(u / u_{\tau}\right)^{2}}\right),
$$

characterized by the positive constants $t_{0}, \kappa$, and $u_{\tau}$. Within the framework of the adiabatic approximation $t_{T}, t_{\phi}<<t_{0}$, the obtained Lorenz system is reduced to the Landau-Khalatnikov equation

$$
t_{0} \dot{u}=-\partial V / \partial u \text {. }
$$

Its form is determined by the effective potential

$$
V=\frac{1}{2}\left[u^{2}-\theta \ln \left(1+u^{2}\right)\right]+\frac{\kappa \alpha^{2}}{2} \ln \left[1+(u / \alpha)^{2}\right],
$$

where $\theta \equiv \phi_{0} / \Delta h, \Delta h \equiv\left(t_{0} t_{T} A_{u} A_{T}\right)^{-1}$ is the scale defined specific crystallization heat, the quantity $V$ is measured in units of $u_{m}^{2}=A_{\phi} A_{T}$. For small values of $\theta$, the curve of the $V$ vs. $u$ dependence has a monotoni- 
cally increasing shape with its minimum at point $u=0$ that corresponds to the cold crystallization mechanism. At $\theta=\theta_{c}^{0}$ where

$$
\theta_{c}^{0} \equiv 1+\alpha^{2}(\kappa-1)+2 \alpha \sqrt{\kappa\left(1-\alpha^{2}\right)},
$$

a plateau appears, which is transformed (for $\theta>\theta_{c}^{0}$ ) into a minimum corresponding to the velocity $u_{e} \neq 0$ and a maximum at point $u^{m}$ separating a minima which meet to the values $u=0$ and $u=u_{e}$. When the parameter $\theta$ increases still further, the minimum at point $u=u_{e}$ is lowered and the height of barrier at $u=u^{m}$ decreases, vanishing at the critical value $\theta_{c}=1+\kappa$.

The steady-state values of the crystallization-front velocity have the form

$$
\begin{gathered}
u_{e}^{m}=u_{00}\left\{1 \mp\left[1+\left(\frac{\alpha}{u_{00}^{2}}\right)^{2}\left(\theta-\theta_{c}\right)\right]^{1 / 2}\right\}^{1 / 2}, \\
u_{00}^{2} \equiv \frac{1}{2}\left[(\theta-1)-(1+\kappa) \alpha^{2}\right] .
\end{gathered}
$$

If the system's energy increases slowly, the jump from zero to $\sqrt{2} u_{00}$ is observed at point $\theta=\theta_{c}$ and then the value $u_{e}$ increases smoothly. If the parameter $\theta$ goes downward quasi-statically, the crystallization front velocity $u_{e}$ smoothly decreases up to the point, where $\theta=\theta_{c}^{0}$ and $u_{e}=u_{00}$, and then jump-like goes down to zero. The hysteresis of such type takes place only at the presence of energy barrier inherent in a first-order transition and is manifested itself if only the parameter $\alpha=u_{\tau} / u_{m}$ is smaller than unity.

The above analysis shows that the effective potential $V(u)$ has the barrier separating the cold and the explosive crystallization modes. As heat density $\theta$ becomes greater than the critical value $\theta_{c}$, this barrier disappears. Thus, at $\theta<\theta_{c}$, the transition to the explosive crystallization mechanism requires the penetrating of energy barrier and at opposite case it realizes spontaneously. The first of appointed situations takes place in the case when the explosive crystallization is initiated by an external beam. With the increase of the coating thickness, the crystallization heat cannot be absorbed by substrate and parameter $\theta$ increases. This leads to that the value $\theta_{c}$ (at which the function $V(u)$ loses barrier) is reached at the critical film thickness and the system transforms into the explosive crystallization regime spontaneously.

\subsection{Self-Organized Criticality of the Explosive Crystallization Process}

The presented approach does not incorporate the diffusive peculiarities of the crystallization process indicated in experimental data discussion. The simplest model to account the diffusive nature of the 
process is the introducing stochastic sources of the Gaussian character into evolution equations. In this Section, we take into account additive noises of the main modes of the self-consistent system. By this, an increase in the noise intensities causes avalanche emergence even in nondriven systems, where the control parameter noise plays a crucial role. A fluctuation regime of this type corresponds to the case, where a distribution of the order parameter appears in an algebraic form with integer exponent [49]. In order to not being restricted to such a particular case, we introduce a unified Lorenz system with a fractional feedback. This assumption allows us to describe a subcritical regime of the avalanche formation in natural manner.

We now focus on the affect of additive noises of the velocity component $u$, temperature $T$, and the thermodynamic potential $\phi$. To this end, we should add stochastic terms $I_{u}^{1 / 2} \xi, I_{T}^{1 / 2} \xi, I_{\phi}^{1 / 2} \xi$ into corresponding equation of motion of main modes. Considering the simplest case, we define such noises as a white noise.

To get rid off such a restriction, the multiplier $u$ in the nonlinear terms of Lorenz system is supposed to be replaced by power term $u^{a}$, with an exponent $0 \leq a \leq 1$. Taking into account the stochastic additions, one obtains the basic equations in dimensionless form

$$
\begin{aligned}
& \dot{u}=-u+v+\sqrt{I_{u}} \xi(t), \\
& \varepsilon \dot{T}=-T+u^{a} \phi+\sqrt{I_{T}} \xi(t), \\
& \delta \dot{\phi}=\left(\phi_{0}-\phi\right)-u^{a} T+\sqrt{I_{\phi}} \xi(t) .
\end{aligned}
$$

It can be seen that the agreement of the Lorenz self-organization scheme with SOC conception, related to self-similar systems is achieved, if one assumes that both positive and negative feedbacks are fractional. Within such a supposition, the adiabatic approximation $\varepsilon, \delta<<1$ leads to the Langevin equation

$$
\dot{u}=f_{a}(u)+\sqrt{I_{a}(u)} \xi(t),
$$

where the force $f_{a}(u)$ and the noise intensity $I_{a}(u)$ are as follows:

$$
\begin{aligned}
& f_{a}(u) \equiv-u+\phi_{0} u^{a} d_{a}(u), \\
& I_{a}(u) \equiv I_{u}+\left(I_{T}+I_{\phi} u^{2 a}\right) d_{a}^{2}(u), \quad d_{a}(u) \equiv\left(1+u^{2 a}\right)^{-1} .
\end{aligned}
$$

The corresponding distribution

$$
P_{a}(u)=\frac{Z^{-1}}{I_{a}(u)} \exp \left\{-E_{a}(u)\right\},
$$

with a partition function $Z$, is determined by an effective potential 


$$
E_{a}(u) \equiv-\int_{0}^{u} \frac{f_{a}\left(u^{\prime}\right)}{I_{a}\left(u^{\prime}\right)} d u^{\prime}
$$

The extremum points of this distribution are determined by the equation

$$
2 a I_{\phi} u^{2 a}+\left(1+u^{2 a}\right)^{2} u^{1-a}\left[\phi_{0}-u^{1-a}\left(1+u^{2 a}\right)\right]=2 a\left(I_{\phi}-2 I_{T}\right),
$$

according to which, the boundary of the flux state

$$
I_{\phi}=2 I_{T},
$$
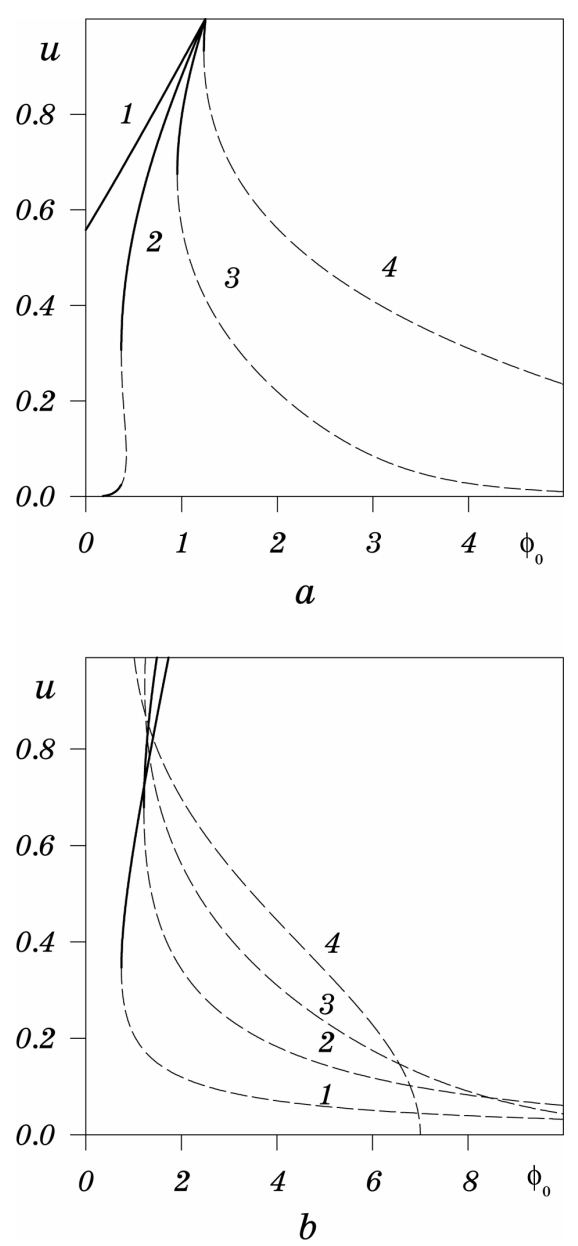

Fig. 13. The $\phi_{0}$-dependence of the steady-state velocity $u$ : $(a)$ at $a=0.75$, $I_{T}=1$ (curves $1-4$ are related to $I_{s}=1,2,3,5$ ); $(b)$ at $I_{T}=1, I_{s}=5$ (curves $1-4$ are related to $a=0.25,0.5,0.75,1.0$. 


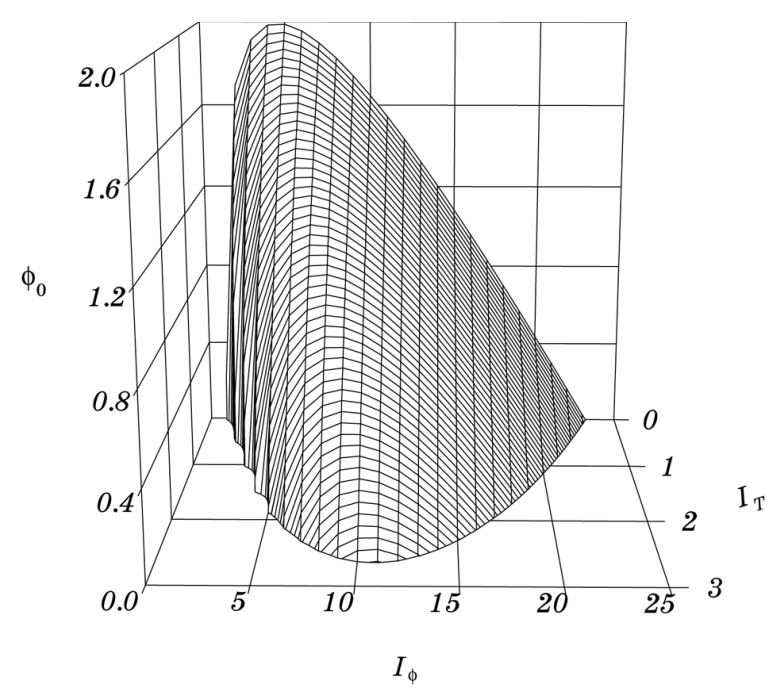

Fig. 14. Three-dimensional phase diagram (the non-avalanche domain is located under the surface).

relates to the condition $u=0$. Critical values of state parameters are fixed by the condition $\left|d u / d \phi_{0}\right|=\infty$ leading to additional equation

$$
\begin{gathered}
u^{2(1-a)}\left(1+u^{2 a}\right)^{2}\left[\left(2+a^{-1}\right)+\left(a^{-1}-1\right) u^{-2 a}\right]- \\
-\frac{1}{2} \phi_{0} u^{1-a}\left(1+u^{2 a}\right)\left[\left(3+a^{-1}\right)+\left(a^{-1}-1\right) u^{-2 a}\right]=2 a I_{\phi} .
\end{gathered}
$$

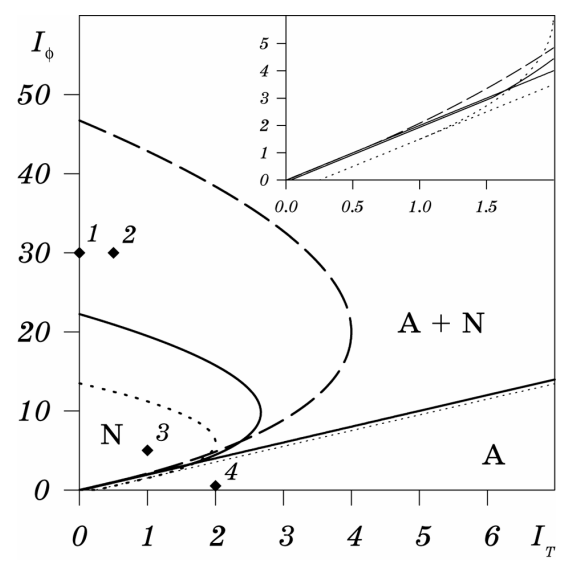

Fig. 15. Phase diagram for the system with $\phi_{0}=0$ and $I_{s}, I_{T} \neq 0$ at $a=0.5$, $0.75,1.0$ (dashed, solid and dotted curves, respectively). Diamonds are related to curves 1-4 in Fig. 18. 


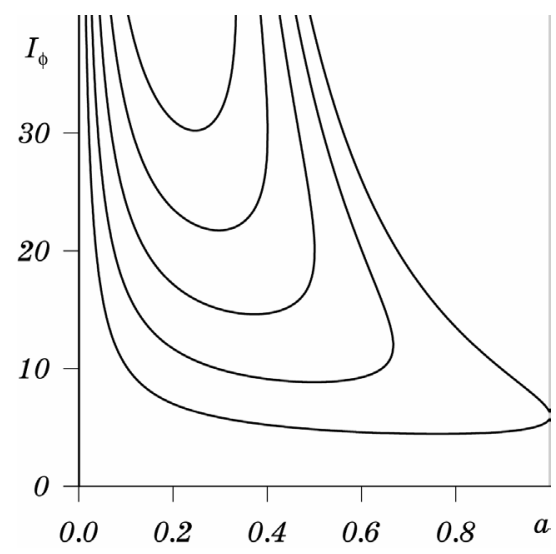

Fig. 16. Phase diagram in the $I_{\phi}-a$ plane at $I_{T}=2,3,4,5,6$ from bottom to top (the non-avalanche domain is located inside the curves).

Above expressions show that an increase in the noise intensity $I_{T}$ causes monotonic $u$-growth, whereas $I_{\phi}$-increase leads to an effective barrier formation near the point $u=0$, so that the dependence $u\left(\phi_{0}\right)$ becomes non-monotonic at magnitudes $I_{\phi}$ above the straight line (3.12) (see Figs. 13). Here, lower branches of curves correspond to unstable magnitudes of the order parameter, while the upper meet the stable ones. According to Fig. 14, the domain, at which avalanches cannot be created, is located near intermediate magnitudes of the state parameters $\phi_{0}, I_{T}, I_{\phi}$. The phase diagram related to the avalanche formation reveals the same form as for the simplest case $a=1$, but the straight-line shifts abruptly to (3.12) with escaping the point $a=1$ (see Fig. 15).

According to Fig. 16, an increase of the noise of the temperature $I_{T}$ increases the domain of the avalanche formation.

\subsubsection{Nonextensive Statistics of Avalanches Ensemble}

In contrast to the previous discussion, where the process of a single avalanche formation has been considered, now we will study analytically the self-similar size distribution of an avalanche ensemble. To this end, we will account noises of a complete set of degrees of freedom, on the one hand, and the fractional feedback type introduced in Section 3.2, on the other one.

Thereby, the Lorenz system unified in the above manner is the basis of our examination. However, the system under consideration is now parameterized by a set of pseudo-thermodynamical variables, which describes the avalanche ensemble in the spirit of the famous Edwards paradigm [13, 14] generalized to nonstationary system 
[49]. With this method, we study time dependences of the avalanche size (density of equipped sites in the avalanche), nonextensive complexity and nonconserved energy of equipped sites in the process of crystallization. Within the framework of the usual synergetic approach, these degrees of freedom play the role of order parameter, conjugate field and control parameter, respectively.

It is principally important that the use of the slaving principle of both synergetics and fractional nature of the system feedback is shown to stipulate the multiplicative character of noise. It will be shown that this causes a nonextensivity of the applied thermodynamical scheme, so that we have to use $q$-weighted averages instead of usual ones. So, energy of equipped sites in the process of crystallization is defined by the expression

$$
\zeta_{q} \equiv \sum_{i} \zeta_{i} p_{i}^{q},
$$

where $p_{i}$ is a probability to enable the site $i$ with energy $\zeta_{i}, q \neq 1$ is a positive parameter that is a measure for the system's nonextensivity determined below. Nonextensive complexity of equipping sites is an analogue to Tsallis entropy [15] that is determined as follows:

$$
\Sigma_{q} \equiv-\frac{\sum_{i} p_{i}^{q}-1}{q-1} .
$$

The three-parameter set of the standard synergetic scheme [2] is completed by the avalanche size $s$.

Following the above-elaborated line, we postulate that a selfconsistent behaviour of the system at issue is presented adequately by a set of pointed out quantities governed by the Lorenz-type equations

$$
\begin{aligned}
& t_{s} \dot{s}=-s+a_{s} \Sigma_{q}+\sqrt{I_{s}} \xi(t), \\
& t_{\Sigma} \Sigma_{q}=-\Sigma_{q}+a_{\Sigma} s^{\tau / 2} \zeta_{q}+\sqrt{I_{\Sigma}} \xi(t), \\
& t_{\zeta} \zeta_{q}=\left(\zeta^{0}-\zeta_{q}\right)-a_{\zeta} s^{\tau / 2} \Sigma_{q}+\sqrt{I_{\zeta}} \xi(t) .
\end{aligned}
$$

Here $t_{s}, t_{\Sigma}, t_{\zeta}$ note relaxation times of corresponding values, $a_{s}, a_{\Sigma}$, $a_{\zeta}$ are related feedback parameters, $I_{s}, I_{\Sigma}, I_{\zeta}$ are respective noise intensities, $\tau$ is a positive exponent and $\zeta^{0}$ is the externally driven energy of the sand motion. The distinguishing feature of the first of these equations is that in a noiseless case genuine characteristics $s, \Sigma_{q}$ are linearly connected. On the other hand, the two last equations (3.16) show that the connection of values $\zeta_{q}, \Sigma_{q}$ (which are of a thermodynamic type) with the avalanche size $s$ is nonlinear. Physically, this means a linear relation between the complexity and the avalanche size near steady state. Moving away, this leads to negative feedback of the 
avalanche size and the complexity on the energy that, in accordance with Le Chatelier principle, results in the energy decrease. Moreover, positive feedback appears between the avalanche size and the energy on the complexity, which causes complexity increase, that is the reason for the avalanche ensemble's self-organization.

To analyze the system (3.16), it is convenient to measure the time $t$ in unit $t_{s}$ and introduce the scales for variables $s, \Sigma_{q}, \zeta_{q}, I_{s}, I_{\Sigma}$, and $I_{\zeta}$ as follows:

$$
\begin{array}{ll}
s^{s c} \equiv\left(a_{\Sigma} a_{\zeta}\right)^{-\frac{1}{\tau}}, & \Sigma_{q}^{s c} \equiv a_{s}^{-1}\left(a_{\Sigma} a_{\zeta}\right)^{-\frac{1}{2}}, \quad \zeta_{q}^{s c} \equiv\left(a_{s} a_{\Sigma}\right)^{-1} ; \\
I_{s}^{s c} \equiv\left(a_{\Sigma} a_{\zeta}\right)^{-1}, \quad I_{\Sigma}^{s c} \equiv a_{s}^{-2}\left(a_{\Sigma} a_{\zeta}\right)^{-1}, \quad I_{\zeta}^{s c} \equiv\left(a_{s} a_{\Sigma}\right)^{-2} .
\end{array}
$$

Then, the scaled Lorenz system (3.16) takes the simple form

$$
\begin{aligned}
& \dot{s}=-s+\Sigma_{q}+\sqrt{I_{s}} \xi(t), \\
& \vartheta \Sigma_{q}=-\Sigma_{q}+s^{\tau / 2} \zeta_{q}+\sqrt{I_{\Sigma}} \xi(t), \\
& \theta \zeta_{q}=\left(\zeta^{0}-\zeta_{q}\right)-s^{\tau / 2} \Sigma_{q}+\sqrt{I_{\zeta}} \xi(t),
\end{aligned}
$$

with the ratios of relaxation times

$$
\vartheta \equiv t_{\Sigma} / t_{s}, \quad \theta \equiv t_{\zeta} / t_{s}
$$

It is worth to notice that the system (3.18) is passed to the form of Eqs. (3.6) if the values $s, \Sigma_{q}, \zeta_{q}, \tau / 2, \vartheta$, and $\theta$ are replaced by $u, T, \phi, a, \varepsilon$, and $\delta$, respectively.

It is well known that a complete set of SOC systems can be reduced to one of two families [50]: systems with deterministic dynamics extremely driven by a random environment (growing interface models, Bak-Sneppen evolution model, etc.) and the stochastic dynamics family (models of earthquakes, forest-fire, etc.). A remarkable peculiarity of the obtained system (3.18) is the possibility to present both mentioned families in a natural manner. The former is related to the noiseless case, when $I_{s}, I_{\Sigma}, I_{\zeta}=0$ but the magnitude of the energy relaxation time is larger than that of the complexity and avalanche size $\left(t_{\zeta} \geq t_{\Sigma}, t_{s}\right)$; on the other hand, a parameter of the environment drive $\zeta^{0}$ has to take a larger value than the critical one $\zeta_{c}=1$ [51]. In such a case, the system (3.18) describes a strange attractor that may represent the behaviour of SOC systems of the first type. A proper stochastic behaviour is relevant for nonvanishing to nonzero noise intensities $I_{s}, I_{\Sigma}, I_{\zeta} \neq 0$ that make possible the appearance of the SOC regime even in the absence of a driven affect $\left(\zeta^{0}=0\right)$.

We will restrict ourselves to the treatment of the stochastic system, where the adiabatic conditions $\vartheta, \theta<<1$ are applicable. Then, the two last equations of the system (3.18) lead to the following dependences: 


$$
\Sigma_{q}(t)=\bar{\Sigma}_{q}+\tilde{\Sigma}_{q} \xi(t), \quad \zeta_{q}(t)=\bar{\zeta}_{q}+\tilde{\zeta}_{q} \xi(t),
$$

where the deterministic and the fluctuating components are determined as follows

$$
\begin{aligned}
& \bar{\Sigma}_{q} \equiv \zeta^{0} s^{\tau / 2} d_{\tau}(s), \quad \tilde{\Sigma}_{q} \equiv \sqrt{I_{\Sigma}+I_{\zeta} s^{\tau}} d_{\tau}(s) \\
& \bar{\zeta}_{q} \equiv \zeta^{0} d_{\tau}(s), \quad \tilde{\zeta}_{q} \equiv \sqrt{I_{\zeta}+I_{\Sigma} s^{\tau}} d_{\tau}(s), d_{\tau}(s) \equiv\left(1+s^{\tau}\right)^{-1} .
\end{aligned}
$$

Due to the slaving principle of synergetics, the initially adiabatic noises of both complexity and energy are transformed to a multiplicative form. On the other hand, the relation between the complexity and energy,

$$
\bar{\Sigma}_{q}=\sqrt{\overline{\zeta_{q}}\left(\zeta^{0}-\bar{\zeta}_{q}\right)},
$$

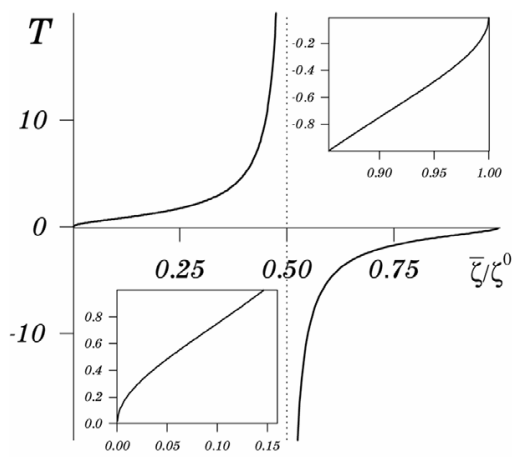

$a$

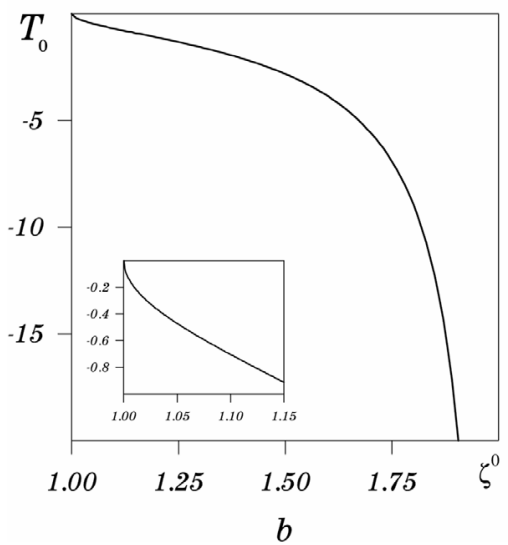

Fig. 17. The energy dependences of the avalanche ensemble temperatures: (a) nonstationary magnitude $T$ versus ratio $\overline{\zeta_{0}} / \zeta^{0} ;(b)$ stationary temperature $T_{0}$ versus $\zeta^{0}$. 
deduced from the dependences (3.21), leads to the expression

$$
T=-\left(1-\frac{\zeta^{0}}{2 \bar{\zeta}_{q}}\right)^{-1} \sqrt{\frac{\zeta^{0}}{\bar{\zeta}_{q}}-1}
$$

for the effective temperature $T \equiv \partial \bar{\zeta}_{q} / \partial \bar{\Sigma}_{q}$. As depicted in Fig. 17, $a, T$ is a monotonically increasing function of the energy with boundary values $T\left(\bar{\zeta}_{q}=0\right)=0$ and $T\left(\zeta_{q}=\zeta^{0} / 2\right)=\infty$. At the latter point, the magnitude $T$ changes instantaneously to $-\infty$ and then increases monotonically again to initial value $T=0$ at $\bar{\zeta}_{q}=\zeta^{0}$. This means that in the domain $0 \leq \bar{\zeta}_{q}<\zeta^{0} / 2$ the avalanche system is dissipative and behaves in usual manner; on the contrast, in the domain $\zeta^{0} / 2<\bar{\zeta}_{q} \leq \zeta^{0}$ a selforganization process evolves, so that an energy increase leads to a complexity decrease, in accordance with a negative temperature. At steady state, where an avalanche has a stationary size $s_{0}=\sqrt{\zeta^{0}}-1$, the temperature takes the stationary value

$$
T_{0}=-\frac{\sqrt{\zeta^{0}-1}}{1-\zeta^{0} / 2}
$$

which is negative in the supercritical domain $1 \leq \zeta^{0}<2$. According to Fig. 17, $b$, the magnitude $T_{0}$ decreases monotonically with the driven energy from value $T_{0}\left(\zeta^{0}=1\right)=0$ to $T_{0}\left(\zeta^{0} \rightarrow 2\right) \rightarrow-\infty$.

The presented self-organization regime relates to externally driven systems, which are relevant for the usual phase transition but not to the SOC itself. To study the latter within the above consideration, let us combine Eqs. (3.20), (3.21) with the first of the equations (3.18) in that way that has been used above for obtaining the Langevin equation. By analogy with Section 3.2, this leads to stochastic equation (3.7), where the effective force and noise intensity are given by Eqs. (3.8) with accuracy to the replacements mentioned after Eqs. (3.19): the quantities $s, \Sigma_{q}, \zeta_{q}, \tau / 2$ have to be taken instead of $u, T, \phi, a$, respectively. Then, all results obtained in Section 3.2 can be used immediately. Particularly, it is found that the influence of a random scattering of the avalanche size is non-essential, whereas energy and complexity noises lead to a crucial effect. The related picture is presented by Fig. 15 taken in plane $I_{\zeta}-I_{\Sigma}$ that is formed by corresponding noise intensities of the avalanche ensemble. The mixed domain $\mathbf{A}+\mathbf{N}$ with respect to the intermittency regime is bounded by the straight line (3.12) and the bell-shaped curve.

According to Fig. 16, where exponent $a$ has to be replaced by $\tau / 2$, the random scattering growth of the complexity extends the SOC domain along the axis of the exponent $\tau$.

Now, we will discuss the distribution of the avalanche size on basis of equations (3.8)-(3.10). For arbitrary noise intensities one has 


$$
\begin{aligned}
& P(s)=\frac{Z^{-1}}{I(s)} \exp \left\{\int_{0}^{s} \frac{f\left(s^{\prime}\right)}{I\left(s^{\prime}\right)} d s^{\prime}\right\} \\
& f(s) \equiv-s+\zeta^{0} s^{\tau / 2} d_{\tau}(s), \\
& I(s) \equiv I_{s}+\left(I_{\Sigma}+I_{\zeta} s^{\tau}\right) d_{\tau}^{2}(s), \quad d_{\tau}(s) \equiv\left(1+s^{\tau}\right)^{-1} .
\end{aligned}
$$

In the SOC regime, the driven energy is vanished, $\zeta^{0}=0$, and the distribution (3.25) behave as depicted in Fig. 18 for different noise intensities of both energy and complexity. It can be seen that the power-law dependence inherent in the SOC regime is observed only in the limits $s<<1$ and $I_{s}, I_{\Sigma}<<I_{\zeta}$. In this case, the distribution (3.25) is reduced to the form

$$
P(s)=\frac{d_{\tau}^{-2}}{Z} \exp \left\{-I_{\zeta}^{-1} \int_{0}^{s} \frac{d_{\tau}^{-2}\left(s^{\prime}\right)}{\left(s^{\prime}\right)^{\tau-1}} d s^{\prime}\right\}, d_{\tau}(s) \equiv\left(1+s^{\tau}\right)^{-1} .
$$

It is easy to see that the deviation of this multiplier from a constant value is estimated with term $\sim s^{2-\tau}$, which increases with decrease of $\tau$ and growth of avalanche size to extremely large magnitudes $s \sim 1$, i.e. with escaping SOC domain. This is confirmed by Fig. 19, where the deviation $\delta \tau$ of the slope of dependence $P(s)$ (Eqs. (3.25)) in the linear domain on the theory parameter $\tau$ is depicted as a function of the parameter $\tau$ itself. In accordance with the above estimation, it can be seen that the deviation $\delta \tau$ takes a maximal value $\delta \tau<10^{-1} \tau$ at non-essential magnitudes $\tau<1$ or, with noise intensity growth to enormous values $I_{s} \sim 10^{3}$.

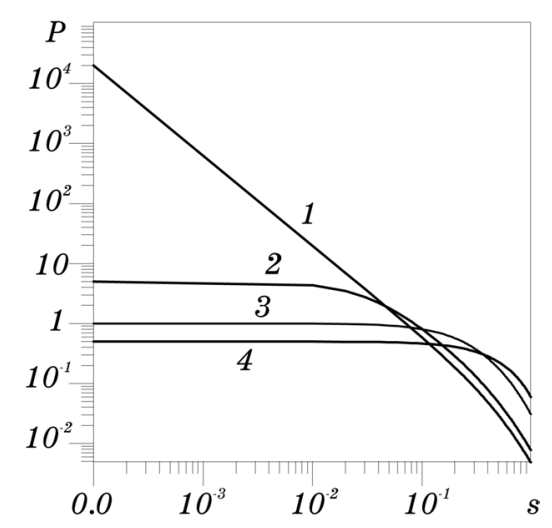

Fig. 18. Distribution function (3.25) at $\tau=1.5$ and regimes pointed out by diamonds in Fig. 15: $1-I_{T}=0, I_{\phi}=50(\mathrm{SOC}) ; 2-I_{T}=0.5, I_{\phi}=30(\mathrm{~A}+\mathrm{N}) ; 3-$ $I_{T}=1, I_{\phi}=5(\mathrm{~N}) ; 4-I_{T}=I_{\phi}=0.5(\mathrm{~A})$. 


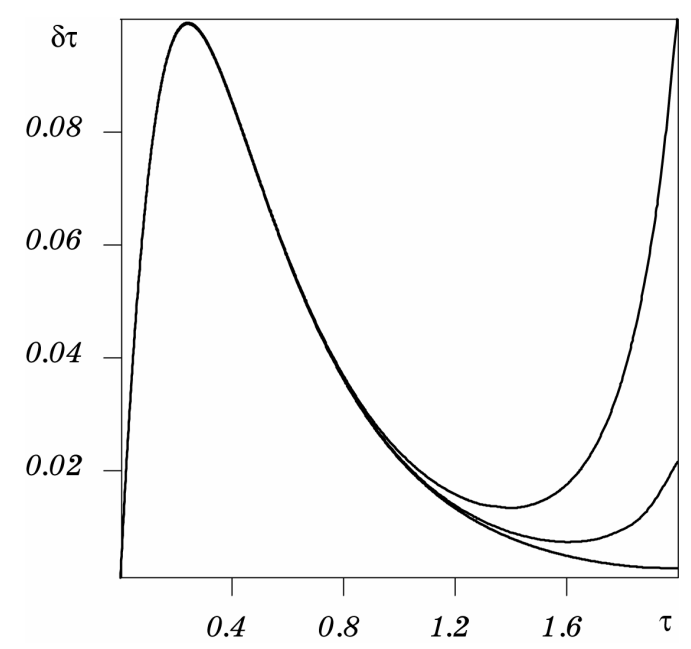

Fig. 19. Deviation $\delta \tau$ of the linear slope of curve 1 depicted in Fig. 18 from parameter $\tau$ versus the exponent $\tau$ itself $\left(I_{\phi}=10,50,10^{3}\right.$ from bottom to top).

Computer simulations. In order to perform the computer simulations, one should note that at $a>1 / 2$ the absorbing state takes

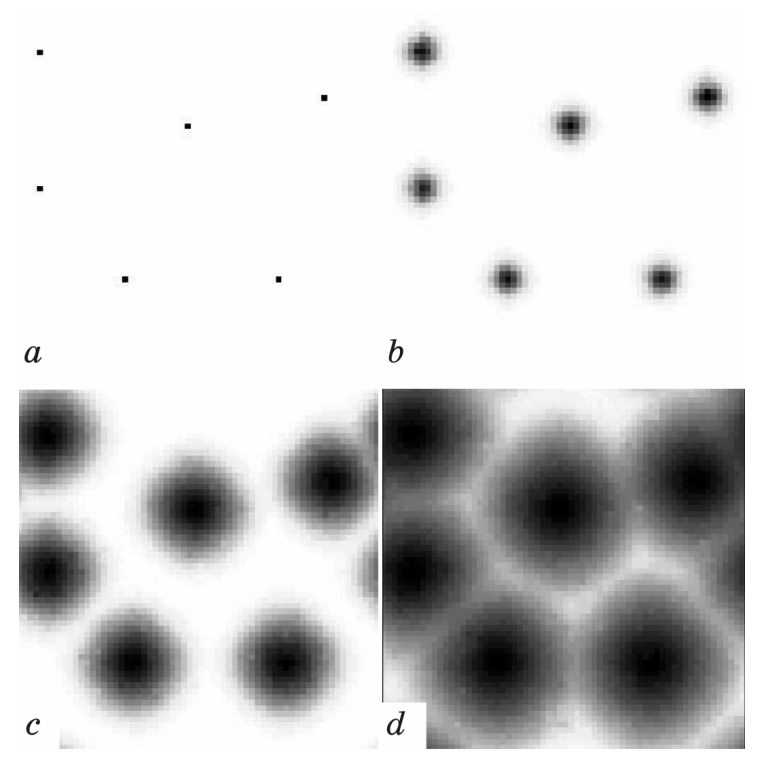

Fig. 20. Computer simulation of the explosive crystallization process (back points correspond to equipped sites). Figures plotted at $a=0.8, D=1.0$, $\Theta=2.0: a, b, c, d$ correspond to $t=0.0,0.005,1.2,2,2.3$. 
place. This state cannot be found considering the most probable values of stochastic variable $s$, it is defined through the transformation of the boundary $s \rightarrow 0$ of the diffusion process $s(t)$. However, obtained results are in good correspondence with well-known laws inherent to the SOC regime.

To examine our model on the $2 D$ grid we introduce the Laplacian into the first equation of the system (3.18). In the adiabatic approximation, we have evolution pictures of the density of equipped sites shown in Fig. 20. It is seen that the diffusion in $r$-space excites the neighbour sites, which transit the system to the crystallized phase (cf. Fig. 12). A special attention can be paid to consideration of the influence of the spatial coupling. Here, spatial coupling promotes an escape from the absorbing configuration where system has been trapped.

By proving so, we plot a fraction $R$ of sites in absorbing configuration in Fig. 21. It is seen that at $D \rightarrow 0$ the system is in absorbing state if $a>1 / 2$. An increase in $D$ or promotes excitation processes which transform all inactive sites (being in absorbing state initially) into active phase (crystallization). Moreover, the process of crystallization has a short time of transformation of absorbed (inactive) sites into the active phase: during only $t \sim 3$ time units, it corresponds to the explosive behaviour of crystallization. It can be seen that the fraction $R$ decreases at $a \cong 3 / 4$ which corresponds to the index $\tau \cong 3 / 2$ where the SOC regime is observed. A further increase in $a$ transforms the system into absorbing state.

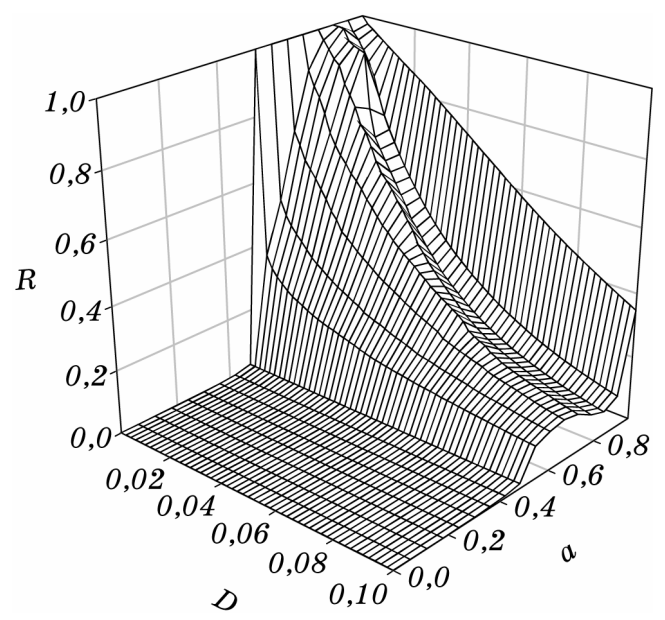

Fig. 21. Fraction of sites in absorbing configuration with $s=0$ : dependences $R(a)$ are shown at $D=0.01, \Theta=0.5 ; D=0.01, \Theta=0.1 ; D=0.06$, $\Theta=1.0$ (curves $1,2,3$ ). 


\subsubsection{Linkage between the Anomalous Diffusion Process and Self-Organized Criticality}

A remarkable peculiarity of expression (3.26) is that, within the limits of $s<<1, I_{s}, I_{\Sigma}<<I_{\zeta}$ inherent in the SOC regime, it can be expressed in terms of standard Gamma-function $\Gamma(x)$ and fractional integral $I_{-s}^{2-\tau}$ of order $2-\tau$ (see Appendix):

$$
P(s)=\frac{d_{\tau}^{-2}}{Z} \exp \left\{-\frac{\Gamma(2-\tau)}{I_{\zeta}} I_{-s}^{2-\tau} d_{\tau}^{-2}(s)\right\} .
$$

On the other hand, it is well-known [16] that expressions of this kind appear as a solution of the fractional Fokker-Planck equation

$$
D_{t}^{\omega} P(s, t)=D_{-s}^{\varpi}\left\{s P(s, t)+\frac{I_{\zeta}}{\Gamma(\varpi)} D_{-s}^{\varpi}\left[d_{\tau}^{2} P(s, t)\right]\right\},
$$

where the fractional derivative $D_{x}^{\omega}$ (see (A.2)) is used to be inverted to the fractional integral (A.1). Multiplying equation (1) by term $s^{2 \pi}$ and averaging over $s$ according to the definitions,

$$
|s| \equiv\left\langle s^{\alpha}\right\rangle^{\frac{1}{\alpha}},\left\langle s^{\alpha}\right\rangle \equiv \int_{-\infty}^{\infty} s^{\alpha} P(s, t) d s, \alpha>0
$$

one obtains at $\alpha \equiv 2 \varpi$

$$
|s|^{z} \sim t, z=\frac{2 \varpi}{\omega}
$$

where $z$ is a dynamical exponent. This relation corresponds to the large time limit, where only the diffusion contribution is essential. Combining expressions (3.27), (3.30) and (A.1) leads to the relations $2-\tau=\varpi=z \omega / 2$, which yield

$$
\tau=2-\frac{z \omega}{2} .
$$

Comparing this equation with the well-known relations for the critical exponents $\tau=1+z / D$ and $\tau=2(1-1 / D)$ corresponding to the process of avalanche formation [50], one obtains

$$
\omega z=\frac{4}{D}
$$

The mean-field magnitudes $\omega=1$ and $D=4$ are related to the dynamical exponent $z=1$ that, in accordance with definition (3.30), is related to the unusual ballistic limit of the SOC regime. On the other hand, the fractional Fokker-Planck equation (3.28) leads to the usual diffusion 
regime with $z=2$ only in the artificial case, when the time-derivative exponent is assumed to be $\omega=1 / 2$.

The obvious reason for such a discrepancy is the non-consistent application of the usual field relations for $\tau$ to the Lorenz system (3.18). In this system, the stochastic degrees of freedom $s, \Sigma_{q}$, and $\zeta_{q}$, whose number is $n=3$, serve as the different space directions. However, the stochastic process evolves for any of these variables in a plane spanned by the given variable itself and its conjugated momentum. Moreover, the multiplicative character of noise, which is determined by the exponent $a$ in expressions (3.8), reduces the fractal dimension of every plane to the value $2(1-a)$ [52]. This result can be achieved considering the temporal behaviour of main statistical moments of a free particle system. As was shown in Ref. [53], where the scaling laws of anomalous behaviour of self-similar system were considered, the multiplicative noise with the amplitude given in a power-law form $x^{a}$ results to the supper-diffusion processes with $x^{2}(t) \propto t^{2 H}, H^{-1}=2(1-a)$. Thus, the resulting fractal dimension of the phase space, in that the stochas-
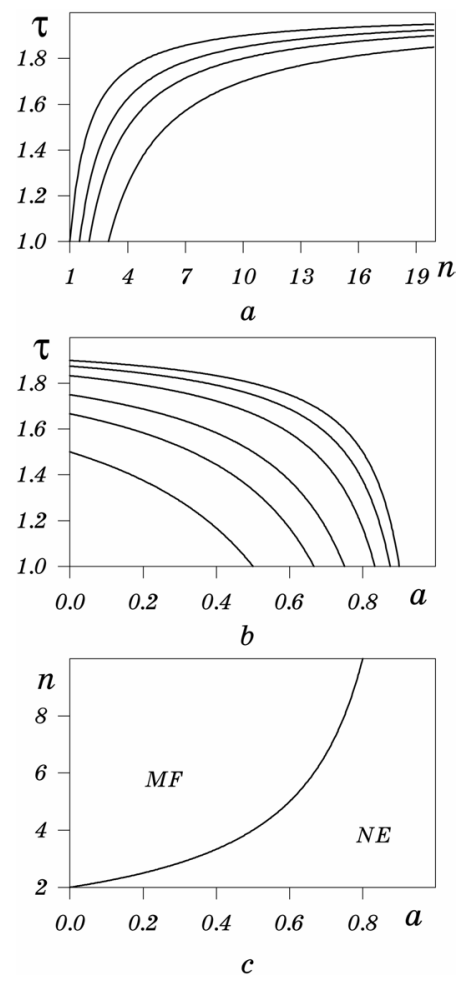

Fig. 22. Dependences of exponent $\tau$ : $(a)$ on equations number $n(a=0,1 / 3$, $1 / 2,2 / 3)$ from top to bottom); (b) on exponent $a(n=2,3,4,6,8,10$ from bottom to top); $(c)$ phase diagram for mean-field and nonextensivity domains. 
tic system evolves, is as follows:

$$
D=2 n(1-a)
$$

where $n=3$ for the used Lorenz system. Inserting this dimension into expression (3.32) leads to $\omega z=2$, which, in contrast to the relation $\omega z=1$ obtained above, is correct in the simplest case $\omega=1, z=2$ [the latter is relevant to a single stochastic degree of freedom $(n=1)$ with additive noise $(a=0)$ ]. In the general case, equations (3.31)-(3.33) yield the final result

$$
\tau=2\left[1-\frac{1}{2 n(1-a)}\right] .
$$

The respective dependences are depicted in Figs. 22, $a, b$ to show that the exponent $\tau$ increases monotonically from its minimum magnitude $\tau=1$ at the critical number $(1-a)^{-1}$ to upper value $\tau=2$ in the limit $n \rightarrow \infty$; thereby, an $a$-growth shifts the dependence $\tau(n)$ to large magnitudes $n$, i.e. decreases the exponent $\tau$.

It is easy to see that relation (3.34) reproduces known results of different approaches for the dimension $D$ (see Ref. [54]). In the case related to mean-field theory, one has $\tau=3 / 2$ and equation (3.34) expresses the number of self-consistent stochastic equations needed for treating the SOC behaviour as a function of the exponent of the corresponding multiplicative noise:

$$
n=\frac{2}{1-a} \text {. }
$$

In accordance with Fig. 22, $c$, a self-consistent mean-field treatment is possible, if the number of relevant equations is larger than the minimum magnitude $n_{c}=2$. Approaches [39-41, 50, 55] represent examples of such considerations, where noise is supposed to have additive character $(a=0)$. Switching the multiplicative noise leads to an $a$-growth and non-contradicting representation of the SOC demands an increase in the number of self-consistent equations: for example, within the field scheme [56] related to directed percolation ( $a=1 / 2)$, the mean-field approximation is applicable for dimensions larger than the critical magnitude $d_{c}=4$; here the Lorenz scheme $(n=3)$ with multiplicative noise is characterized by the exponent $a=1 / 3$ (see below).

Let us now focus on the relation of the above exponents to the nonextensivity parameter $q$ related to Tsallis definitions (3.14), (3.15) [15]. The relevant kinetic behaviour could be described by the nonlinear Fokker-Planck equation

$$
D_{t}^{\omega} P(s, t)=D_{-s}^{2} P^{q}(s, t),
$$


where $D_{t}^{\omega}$ is the fractional derivative and the measure units are chosen in such a way that the effective diffusion coefficient disappears $(\omega>0, q>0$ are the relevant exponents [57-59]). Supposing a normalized distribution function in a self-similar form

$$
P(s, t)=s_{c}^{-1} P(x) ; s_{c} \equiv s_{c}(t), x \equiv s / s_{c},
$$

we obtain

$$
s_{c}^{q+1} \sim t^{\omega}, P^{q-1} \sim x^{2}, x \rightarrow 0 .
$$

On the other hand, we could use the fractional Fokker-Planck equation of the type of Eq. (3.28):

$$
D_{t}^{\omega} P(s, t)=D_{-s}^{2 \varpi} P(s, t) .
$$

Inserting the solution (3.37), one finds the dependences

$$
s_{c}^{2 \varpi} \sim t^{\omega}, P \sim x^{2 \varpi-1},
$$

whose comparison with Eqs. (3.38) yields

$$
q=2 \varpi-1 .
$$

Because the average $|s|$ in Eq. (3.29) is reduced to the scale $s_{c}$ in the case of self-similar systems, the relevant dependences (3.30), (3.38) and (3.40) give

$$
q=z \omega-1
$$

Combining this equality with Eqs. (3.32), (3.33) leads to the resulting expression for the nonextensivity parameter of the considered system:

$$
q=\frac{2}{n(1-a)}-1 \text {. }
$$

The maximum magnitude $q=2 / n-1$ is related to systems with additive noise $(a=0)$, which is relevant to the mean-field picture at a number of governing equations $n<2(n=1)$. Switching the multiplicative noise with increasing exponent $a>0$ leads to a $q$-growth and the self-organizing system gets a nonextensive character $(q \geq 1)$ in the limit $q \leq 1 /(1-a)$ of the mean-field applicability domain. In accordance with the above estimation, the fractional Lorenz system is nonextensive, essentially if the exponent $a>2 / 3$.

It is worth to remember that above we have assumed the superdiffusion process only to be related to Lévy flights at discrete time instant 
with arbitrary displacements, including infinite ones [60]. Related to the Fokker-Planck equation (3.28), such processes are characterized by exponents $\omega=1$ and $\varpi<1$, the first of which is constant, whereas the second one characterizes the fractal time-sequence of the Lévy flights and leads to the dynamical exponent $z \equiv 2 \varpi / \omega<2$ (see the last of equations (3.30)). The probability distribution of the displacement $\mathbf{x}$, that is dependent on microscopic conditions, reads

$$
p(\mathbf{x}) \sim x^{-(D+\gamma)}
$$

and is characterized by the fractal dimension $D$ and the microscopic step exponent $\gamma$. It is obvious that in the case of rare events, when $\gamma<2$, the dynamical exponent $z$ is reduced to the microscopic step exponent $(z=\gamma<2$ ), whereas at $\gamma \geq 2$ one has $z=2$ [61].

In the opposite case of subdiffusion processes, a microscopical ingredient is the random Lévy walks instead of the discrete Lévy flights. This process evolves continuously in course of the time over discrete placed traps, so that the exponent $\omega<1$ describes fractal properties of this space that depend on microscopic conditions. These properties generate the transformation of the usual Boltzmann-Gibbs statistics in a nonextensive manner [15]. The subdiffusion process is presented by the Tsallis-type distribution [62]

$$
p(\mathbf{x}) \propto\left[1+\beta(q-1) x^{2}\right]^{-\frac{1}{q-1}}, \quad \beta=\text { const }>0,
$$

where the deviation $q-1$ of the nonextensive parameter is caused by the fractal nature of the system phase space that is connected to the step exponent $\gamma$ as follows:

$$
q=1+\frac{2}{D+\gamma}
$$

A formal advantage of this distribution (3.45) is that the corresponding $q$-weighted average

$$
\left\langle\mathbf{x}^{2}\right\rangle_{q} \equiv \int \mathbf{x}^{2} p^{q}(\mathbf{x}) d^{D} x
$$

where the integrand varies as $x^{-(1+\gamma)}$, converges for arbitrary step exponents $\gamma>0$. As a result, the equation of motion of the random Lévy walker is given by

$$
\left\langle\mathbf{x}^{2}\right\rangle_{q} \sim t^{\omega}, \quad \omega= \begin{cases}q-1, & \text { at } \gamma, q<2, \\ 1-(q-1) \frac{D}{2}, & \text { at } \gamma \geq 2, q>1 .\end{cases}
$$

In contrast to Eq. (3.40), where the exponents $\varpi<1, \omega=1$ are relevant for the superdiffusion, one here has inverted relations: 
$\varpi=1, \omega<1$. Thus, in accordance with the subdiffusion nature, the last equality (3.30) yields the dynamical exponent $z>2$.

In the general case of $\varpi, \omega \neq 1$, inserting Eqs. (3.48) into the relation (3.42) leads to the result

$$
z=\left\{\begin{array}{llc}
\frac{q+1}{1-\frac{D}{2}(q-1)} & \text { at } & 1<q \leq q_{D}, \\
\frac{q+1}{q-1} & \text { at } & q_{D} \leq q \leq 2,
\end{array}\right.
$$

where the boundary value of the nonextensivity parameter is introduced:

$$
q_{D} \equiv \frac{4+D}{2+D} .
$$

To avoid a mistake, let us focus on Eqs. (3.31), (3.32), which could be related to the real phase space and the configurational one (the latter is spanned by variables of governing equations), the above-obtained relations (3.48)-(3.50) are relevant for the real phase space only. This is reflected by addressing the fractal dimension $D$ to the only real coordinate space in the former case, whereas in the latter it is reduced to the effective value (3.33). According to our treatment, relation (3.33) plays a central role since, in analogy with a renormalization group, we have considered the properties of the configurational space but not real diffusion process.

\section{CONCLUSIONS}

This review is concerned the study of synergetic systems in noisy environment. We addressed our approach to the consideration of selforganization processes during the plastic flow. It was shown that random fluctuation of the media can be a reason to the qualitative reconstruction of condensed matter structure in the course of plastic deformation. We discussed the representation of the explosive crystallization processes, which are familiar to the self-organ-ized criticality regime. At last, the evolution of general type of models to describe the condensed matter is investigated in detail.

Considering the synergetic models with different dimensionalities, we found that the correlation between fluctuations of the freedom degrees of the system plays a crucial role and makes the system to pass from one structure to another through re-entrant phase transitions. Developing the well-known cumulant expansion method, we investigated the character of diffusion of linear and point defects separately within the framework of the mean-field approximation, which gives the qualitative results, and with using computer simulation procedure. Our results are in a good correspondence with a well-known conclusion that the 
reconstruction of macroscopic picture of plastic flow is a result of processes, which occur in microscales where the density of dislocations varies discontinuously. As shown, the discontinuous behaviour of the density of linear defects is explained as a result of cross correlations between fluctuations of different freedom degrees.

The description of the explosive crystallization is given on the basis of the Lorenz-type model. The conditions to the explosive crystallization processes are determined. We showed that the transition to the explosive crystallization has properties inherent to the regime of selforganized criticality. The avalanche-like dynamics is represented for both single avalanche system and an avalanche ensemble. Considering the noise induced transitions we have shown that the absorbing state appeared at large noise intensities can be destroyed introducing the spatial coupling in the system. It was shown that the main laws of selforganized criticality are applicable to the explosive crystallization process. We found main exponents and set relations between dynamical and geometrical characteristics of the system phase space. It was shown that the explosive crystallization is related to the anomalous diffusion.

We are looking forward that the described results, models and assumptions presented in this review could give a stimulus to develop the theory of self-organization of defect structure during the plastic flow. One can expect that obtained results will be of interest for the researches in the field of plastic deformation to predict the resources of details and constructions. The representations of reconstruction of defect structure can be useful to interpret the experimental data in which different defects are observed with increasing deformation or diffusion constant.

Perspectives of the work can be as follows: (i) generalization of the theory of fluctuation reconstruction of phase transition; from mathematical point of view picture of noise induced phase transition need to be established in a more rigorous way to account spatial correlations; (ii) the theory of escaping from absorbing configuration should be developed to investigate the ergodicity breaking effect more precisely; (iii) theory of SOC should be examined introducing the fractional derivatives into motion equation to reflect the anomalous diffusion regime.

\section{APPENDIX}

Here, the basic properties of fractional integral and derivative are presented. The integral of fractional order $\varpi$ is defined by equality $[9,63]$

$$
I_{x}^{\varpi} f(x) \equiv \frac{1}{\Gamma(\varpi)} \int_{0}^{x} \frac{f\left(x^{\prime}\right)}{\left(x-x^{\prime}\right)^{1-\varpi}} d x^{\prime}, \quad \varpi>0
$$


where $f(x)$ is an arbitrary function, $\Gamma(x)$ is the standard Gammafunction. To be inverted to the fractional integral, the relevant derivative $D_{x}^{\varpi} \equiv I_{x}^{-\varpi}$ of order $\varpi>0$ is determined as follows:

$$
D_{x}^{\varpi} f(x) \equiv \frac{1}{\Gamma(-\varpi)} \int_{0}^{x} \frac{f\left(x^{\prime}\right)}{\left(x-x^{\prime}\right)^{1+\varpi}} d x^{\prime} .
$$

In the region $0<\varpi<1$, it is convenient to use the expression

$$
D_{x}^{\varpi} f(x) \equiv \frac{\varpi}{\Gamma(1-\varpi)} \int_{0}^{x} \frac{f(x)-f\left(x^{\prime}\right)}{\left(x-x^{\prime}\right)^{1+\varpi}} d x^{\prime}
$$

where we take into account the known equality $x \Gamma(x)=\Gamma(x+1)$ for $x \equiv-\varpi$.

\section{REFERENCES}

1. G. Nicolis and I. Prigogine, Self-Organization in Nonequilibrium Systems (New York, London, Sydney, Toronto: A Wiley-Interscience Publ., John Wiley \& Sons: 1977).

2. H. Haken, Synergetics - An Introduction (Berlin, Heidelberg, New York: Springer-Verlag: 1983).

3. C. W. Gardiner, Handbook of Stochastic Methods (Berlin: Springer-Verlag: 1983).

4. W. Horsthemke and R. Lefever, Noise-Induced Transitions (Berlin: Springer-Verlag: 1984).

5. J. Garcia-Ojalvo and J. M. Sancho, Noise in Spatially Extended Systems (New York: Springer-Verlag: 1999).

6. P. Bak, How Nature Works: the Science of Self-Organized Criticality (Oxford: Oxford University Press: 1997).

7. H. J. Jensen, Self-Organized Criticality. Emergent Complex Behaviour in Physical and Biological Systems. Cambridge Lecture Notes in Physics (Cambridge: Cambridge University Press: 1998).

8. J. Feder, Fractals (New York: Plenum: 1989).

9. Applications of Fractional Calculus in Physics (Ed. R. Hilfer) (Singapore: World Scientific: 2000).

10. V. V. Rubin, Bol'shie Plasticheskie Deformatsii i Razrushenie Metallov (Large Plastic Deformations and Failure of Metals) (Moscow: Metallurgiya: 1985) (in Russian).

11. V. E. Panin, V. A. Likhachyov, and Yu. V. Grinyayev, Strukturnyye Urovni Deformatsii Tvyordykh Tel (Structural Levels of Deformation of Condensed Matter) (Novosibirsk: Nauka: 1985) (in Russian).

12. V. P. Skripov and V. P. Koverda, Spontannaya Kristallizatsiya Pereokhlazhdyonnykh Zhydkostej (Spontaneous Crystallization of Supercooled Liquids) (Moscow: Nauka: 1984) (in Russian).

13. S. F. Edwards and R. B. S. Oakeshott, Physica A, 157: 1080 (1989).

14. S. F. Edwards, Granular Matter: An Interdisciplinary Approach (Ed. A. Metha) (New York: Springer-Verlag: 1994). 
15. C. Tsallis, Lecture Notes in Physics (Eds. S. Abe and Y. Okamoto) (Heidelberg: Springer-Verlag: 2001).

16. G. M. Zaslavsky, Chaos, 4: 25 (1994); idem, Physica D, 76: 110 (1994); A. I. Saichev and G. M. Zaslavsky, Chaos, 7: 753 (1997).

17. A. Damask and J. Diens, Point Defects in Metals (New York: Gordon and Breach: 1963).

18. J. Friedel, Dislocations (Oxford, London, Edinburg, NewYork, Paris, Frankfurt: Pergamon Press: 1964).

19. J. D. Vishnyakov, Defekty Upakovki v Kristallicheskoj Strukture (Defects of Packing in Crystal Structure) (Moscow: Metallurgiya: 1970) (in Russian).

20. J. P. Hirth and J. Lothe, Theory of Dislocations (New York: McGraw-Hill: 1968).

21. B. I. Smirnov, Dislokatsionnaya Struktura i Uprochnenie Kristallov (Dislocation Structure and Strengthening) (Leningrad: Nauka: 1981) (in Russian).

22. V. I. Vladimirov and A. E. Romanov, Disklinatsii v Kristallakh (Disclinations in Crystals) (Leningrad: Nauka: 1986) (in Russian).

23. R. de Witt, Continual Theory of Disclinations. (Moscow: Mir Publishers: 1977) (Russian translation).

24. A. I. Olemskoi, Physics Reviews (Ed. I. M. Khalatnikov) (London: Gordon \& Breach: 1996), vol. 18, Part 1, p. 1.

25. A. I. Olemskoi and A. V. Khomenko, Usp. Fiz. Met., 2: 189 (2001).

26. S. E. Mangioni, R. R. Deza, R. Toral, and H. S. Wio, Phys. Rev. E, 61: 223, (2000).

27. P. Jung and P. Hänggi, Phys. Rev. A, 35: 4467 (1987); F. Castro, H. S. Wio, and G. Abramson, Phys. Rev. E, 52: 159 (1995).

28. D. O. Kharchenko and I. A. Knyaz', Mettallofiz. Noveishie Tekhnol., 24, No. 3: 389 (2002).

29. E. A. Novikov, JETP, 47: 1919 (1964).

30. M. A. Munoz, Phys. Rev. E, 57: 1377 (1998).

31. A. I. Olemskoi and D. O. Kharchenko, Met. Phys. Adv. Tech., 16: 841 (1996).

32. A. I. Olemskoi and D. O. Kharchenko, Physics of Solid State, 42: 520 (2000).

33. D. O. Kharchenko, Physica A, 308: 101 (2002).

34. D. O. Kharchenko and I. A. Knyaz', Eur. Phys. J. B, 32: 375 (2003).

35. N. G. Van Kampen, Stochastic Processes in Physics and Chemistry (Amsterdam: North Holland: 1992).

36. V. E. Shapiro, Phys. Rev. E, 48: 109 (1993).

37. H. Risken, The Fokker-Planck Equation (Berlin: Springer Verlag: 1989).

38. S. F. Edwards and D. R. Wilkinson, Proc. Roy. Soc. A, 381: 17 (1982).

39. A. Mehta and G. C. Barker, Rep. Prog. Phys., 57: 383 (1994).

40. J. -P. Bouchaud, M. E. Cates, J. R. Prakash, and S. F. Edwards, J. Phys. I (France), 4: 1383 (1994).

41. K. P. Hadeler and C. Kuttler, Granular Matter, 2: 9 (1999).

42. P. Bak and K. Sneppen, Phys. Rev. Lett., 71: 4083 (1993).

43. A. I. Olemskoi, A. V. Khomenko, and V. P. Koverda, Physica A, 284: 79 (2002).

44. L. Pietronero, A. Vespignani, and S. Zapperi, Phys. Rev. Lett., 72: 1690 (1994); idem, Phys. Rev. E, 51: 1711 (1995).

45. V. P. Koverda and V. P. Skripov, Rasplavy, 1, No. 4: 3 (1987) (in Russian).

46. N. M. Bogdanov, V. P. Koverda, V. N. Skokov, V. P. Skripov, and A. A. Dik, Kristallografiya, 33, No. 5: 1251 (1988) (in Russian). 
47. D. A. Kurtze, Phys. Rev. B, 34: 1770 (1986).

48. V. P. Koverda, Zh. Tekhn. Fiz., 64, No. 3: 62 (1994) (in Russian).

49. A. I. Olemskoi, A. V. Khomenko, and D. O. Kharchenko, Physica A, 323: 263 (2003).

50. A. Vespignani and S. Zapperi, Phys. Rev. Lett., 78: 4793 (1997); idem, Phys. Rev. E, 57: 6345 (1998).

51. A. I. Olemskoi and A. V. Khomenko, JETP, 83: 1180 (1996).

52. A. I. Olemskoi, Physics-Uspekhi, 41: 269 (1998).

53. D. O. Kharchenko, Fluct. and Noise Lett., 2, No. 4: L287 (2002).

54. A. Chessa, E. Marinari, A. Vespignani, and S. Zapperi, Phys. Rev. E, 57: R6241 (1998).

55. L. Gil and D. Sornette, Phys. Rev. Lett., 76: 3991 (1996).

56. A. Vespignani, R. Dickman, M. A. Mucoz, and S. Zapperi, Phys. Rev. Lett., 81: 5676 (1998); idem, Phys. Rev. E, 62: 4564 (2000).

57. A. I. Olemskoi, JETP Let., 71: 285 (2000).

58. A. I. Olemskoi and D. O. Kharchenko, Physica A, 293: 178 (2001).

59. D. O. Kharchenko and S. V. Kohan, Eur. Phys. J. B, 29: 97 (2002).

60. J. -P. Bouchaud and A. Georges, Phys. Rep., 195: 127 (1991).

61. H. C. Fogedby, Phys. Rev. E, 58: 1690 (1998).

62. D. H. Zanette and P. A. Alemany, Phys. Rev. Lett., 75: 366 (1995).

63. S. G. Samko, A. A. Kilbas, and O. I. Marichev, Fractional Integrals and Derivatives-Theory and Applications (New York: Gordon and Breach: 1993). 